\title{
A classification of inverse limit spaces of tent maps with a nonrecurrent critical point
}

\author{
BRIAN E RAINES \\ SONJA ŠTIMAC
}

\begin{abstract}
In this paper we prove the nonrecurrent case of the Ingram conjecture by showing that if $T_{s}$ and $T_{t}$ are two tent maps with nonrecurrent critical points then $\lim _{\longleftarrow}\left\{[0,1], T_{s}\right\}$ is homeomorphic to $\lim _{\longleftarrow}\left\{[0,1], T_{t}\right\}$ if and only if $s=t$.
\end{abstract}

37B10; 37B45

\section{Introduction}

Inverse limits of unimodal maps arise naturally in many different settings; see Barge and Martin [7]. They appear in discussions of the topology of one-dimensional nonhyperbolic attractors such as the Hénon attractor as in Barge and Martin [8], Barge and Diamond [3] and Bruin [13]. They arise as substitution tiling spaces (see Anderson and Putnam [1], Barge and Diamond [5] and Barge, Jacklitch and Vago [6]) and recently they have been studied in connection with certain models from macroeconomics (see Kennedy, Stockman and Yorke [22; 21] and Medio and Raines [23; 24]).

A driving problem in the theory of unimodal inverse limit spaces is that of characterizing the inverse limit space in terms of the dynamics of the associated bonding map. In 1992, W T Ingram stated the following conjecture:

Let $T$ and $T^{\prime}$ be tent maps. Then $\lim \{[0,1], T\}$ is homeomorphic to $\lim _{\longleftarrow}\left\{[0,1], T^{\prime}\right\}$ if, and only if, $T=T^{\prime}$.

This conjecture has received a significant amount of attention in the last sixteen years, with many special cases being established. Notably, in 1995 Barge and Diamond [4] proved the Ingram Conjecture in the special case that the tent maps were one of the three maps with a critical point on a period five orbit. Bruin [14] and Swanson and Volkmer in [30] extended their results. In 2003 Kailhofer [19; 20] proved the Ingram conjecture in the special case that the critical orbit is periodic (see also Block, Jakimovik, Kailhofer and Keesling [10]). More recently Štimac [29] proved that the Ingram Conjecture is true in the case that the critical orbit is finite (either periodic or preperiodic) (details for 
the periodic case were given in Štimac's thesis [27]). Thus in the case that $T$ is a tent map with a finite critical orbit, it is known that Ingram's Conjecture is true. Hence for a countable collection of parameter values, Ingram's Conjecture has been verified. These spaces have many features in common. Each of them is locally almost everywhere the product of a Cantor set and an open arc. They each have only finitely many points where this local structure is not present (either at a finite collection of folding points by Bruin [14] in the case that the critical orbit is preperiodic, or a finite collection of endpoints by Barge and Martin [9] in the case that the critical orbit is periodic), and every proper subcontinuum is an arc or a point. A folding point is any point, $x$, in the inverse limit space with the property that its $n$-th coordinate, $x_{n}$, is in $\omega(1 / 2)$ for all $n \in \mathbb{N}$; see Raines [25]. Alternatively, $x$ is a folding point in the inverse limit space provided there is no neighborhood of $x$ that is homeomorphic to the product of a zero-dimensional set and an arc.

Several papers have also been written studying the structure of these inverse limits without the assumption that the critical orbit is finite; see Brucks and Bruin [11], Bruin [15], Good, Knight and Raines [17] and Good and Raines [18] for the case that the critical orbit is not dense, and see Barge, Brucks and Diamond [2] for the case that the critical orbit is dense. A natural subdivision of the remaining case of the Ingram Conjecture is into the case that the critical point is recurrent and the case that the critical point is nonrecurrent. In the case that the critical point is recurrent the topology of the inverse limit can be quite complicated. It can have subcontinua like $\sin 1 / x$ curves or other tent map inverse limit spaces [11] or it can even have the property that every neighborhood contains a copy of every tent map inverse limit space [2].

We consider the case that the critical point is nonrecurrent but has an infinite orbit. This implies that the bonding map is long-branched and that critical $\omega$-limit set is infinite. Moreover these spaces have only arcs and points as proper subcontinua, but they have infinitely many folding points and only one endpoint. The collection of inverse limit spaces generated by tent maps with a nonrecurrent critical point includes the tent map inverse limits with countably many folding points (such as those described in [17]) and also many with an uncountable collection of folding points (such as those described in [18]).

\subsection{Our approach}

In the rest of the paper we adopt a symbolic description of these inverse limit spaces, and we use the symbolic description solely in all of our proofs. For that reason in this subsection we give a brief heuristic account of our approach to the proof of Ingram's Conjecture. We use this symbolic approach to describe some of the structures found in composants of these spaces in [28] and [26] which we use extensively in this paper. 
Let $s, t \in(\sqrt{2}, 2]$ be two parameters such that $T_{s}$ and $T_{t}$ are tent maps with nonrecurrent and non-preperiodic critical points. Let $K_{s}$ denote $\lim \left\{[0,1], T_{s}\right\}$ and let $K_{t}$ denote

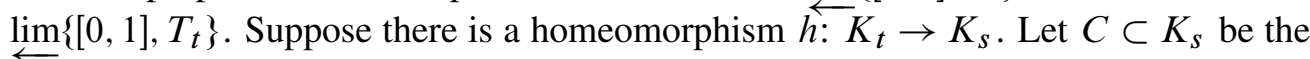
composant of $K_{s}$ which contains the unique endpoint $(\ldots, 0,0,0)$ for $K_{s}$, and let $C^{\prime} \subset K_{t}$ be the composant which contains the unique endpoint $(\ldots, 0,0,0)$ for $K_{t}$. Then $h\left(C^{\prime}\right)=C$.

We let $\mathbb{N}$ stand for the positive integers and $\mathbb{Z}_{+}=\mathbb{N} \cup\{0\}$. We begin by describing countably many coordinate schemes on each of these composants. We define them by, given $p \in \mathbb{Z}_{+}$,

$$
E_{p}=C \cap\left(\bigcup_{n=p}^{\infty} \pi_{n}^{-1}(1 / 2)\right)
$$

and given $q \in \mathbb{Z}_{+}$,

$$
E_{q}^{\prime}=C^{\prime} \cap\left(\bigcup_{n=q}^{\infty} \pi_{n}^{-1}(1 / 2)\right) .
$$

We call the points in $E_{p}$ p-points. Similarly, we call points in $E_{q}^{\prime} q$-points. Given $p, q \in \mathbb{Z}_{+}$, then each collection can be stratified into levels by saying, for $x \in E_{p}$, the $p$-level of $x$ is $L_{p}(x)=k$ if, and only if, $x \in \pi_{p+k}^{-1}(1 / 2)$ and, for each $x^{\prime} \in E_{q}^{\prime}$, the $q$-level of $x^{\prime}$ is $L_{q}\left(x^{\prime}\right)=k$ if, and only if, $x^{\prime} \in \pi_{q+k}^{-1}(1 / 2)$, where $k \in \mathbb{Z}_{+}$. Notice that each set, $\pi_{p+k}^{-1}(1 / 2)$ is a Cantor set plus a countable set (due to the inclusion of the composant $C$ ) of points in $K_{s}$; however we restrict this set to only the one endpoint-composant where it is a countable collection of points that are isolated with respect to the "arclength" topology on that composant.

We then describe "canonical" chainings of these inverse limits in terms of cylinder sets given by the symbolic representation of the space. Each of these chainings have two parameters, ie, for $p \in \mathbb{Z}_{+}$and $n \in \mathbb{N}$ we have a chaining $\mathcal{C}_{p, n}$. We show that given a chaining $\mathcal{C}_{p, n}$ of $K_{s}$, there is a $q \in \mathbb{Z}_{+}, m \in \mathbb{N}$, and a chaining $\mathcal{C}_{q, m}^{\prime}$ of $K_{t}$, such that $h\left(\mathcal{C}_{q, m}^{\prime}\right)$ refines $\mathcal{C}_{p, n}$ and such that every one of our coordinate points in $C^{\prime}$ with $q$-level greater than zero is mapped into an arc component of a link of $\mathcal{C}_{p, n}$ which contains a unique coordinate point in $C$ with $p$-level greater than zero. This allows us to "redefine" the homeomorphism $h: K_{t} \rightarrow K_{s}$ to a map $h_{q, p}: C^{\prime} \rightarrow C$ that is "pseudohomotopic" to $h$. We accomplish this by "scooting" the image of the $q$-points with $q$-level greater than 0 to line up precisely with the $p$-points which have $p$-level greater than 0 . We then extend the map between the $q$-points in a monotone manner to get $h_{q, p}$ defined on the entire composant $C^{\prime}$, and then show that $h_{q, p}$ is both injective and surjective. Since our main concern for $h_{q, p}$ is that it maps $q$-points to $p$-points in a regimented manner, we do not check if there is a homotopy $H: C \times[0,1] \rightarrow C$ from $h$ to $h_{q, p}$. But the construction of $h_{q, p}$ is reminiscent of a homotopic transformation of $h$. 
For this reason we informally refer to $h_{q, p}$ as a "pseudohomotopic to $h$ " rather than homotopic. We also show that $h_{q, p}$ maps all the $q$-points in $C^{\prime}$ with the same $q$-level to $p$-points in $C$ with the same $p$-level. This allows us to show that, restricted to our coordinate scheme, $h_{q, p}$ is a conjugacy between the induced shift homeomorphism on $K_{t}$ and some iterate of the induced shift homeomorphism on $K_{s}$. This leads then to the proof of our Main Theorem: $K_{s}$ and $K_{t}$ are not homeomorphic if $s \neq t$.

\subsection{Outline}

In Section 2 we collect the preliminary definitions and background information required for the rest of the paper. We recall the symbolic representation of these inverse limit spaces given by Brucks and Diamond [12]. We give our definitions of the collection of identification points (which we call $p$-points or $q$-points depending on the context), and of canonical chainings (which we call $\mathcal{C}_{p, n}$ ).

In Section 3 we begin a description of the structure of the composant containing the endpoint related to these chainings. We follow that with a discussion of how $h$ maps the identification points, and we construct a map $h_{q, p}$ that is "pseudohomotopic" to $h$ but that sends our identification points from $K_{t}$ to identification points of $K_{s}$ and is monotone between these identification points. We show that $h_{q, p}$ is a conjugacy between the induced shift maps restricted to the collection of identification points in the composant containing the end point. Then we use this fact to prove our Main Theorem.

We end the paper with a technical Appendix where we collect many of the results regarding the finer structure of the endpoint composant.

\section{Preliminaries}

Let $\mathbb{N}$ be the set of natural numbers $\{1,2,3, \ldots\}$ and $\mathbb{Z}_{+}$be the set of nonnegative integers $\{0,1,2, \ldots\}$. We consider the family of tent maps parameterized by $s \in$ $(\sqrt{2}, 2]$,

$$
T_{s}(x)= \begin{cases}s x, & \text { if } 0 \leq x \leq 1 / 2 ; \\ s(1-x), & \text { if } 1 / 2 \leq x \leq 1\end{cases}
$$

with critical point $1 / 2$. Let $K_{s}$ denote the inverse limit of $T_{s}$, ie,

$$
K_{s}=\lim _{\longleftarrow}\left\{[0,1], T_{s}\right\}=\left\{\left(\ldots, \xi_{-3}, \xi_{-2}, \xi_{-1}\right) \in[0,1]^{\mathbb{N}}: \xi_{-i}=T_{s}\left(\xi_{-i-1}\right)\right\} .
$$

Throughout this paper we assume that the parameters are chosen so that the maps we consider have a nonrecurrent critical point, ie, $1 / 2 \notin \omega(1 / 2)$. This implies, among other things, that the tent maps we consider are long-branched: 
Definition 2.1 The map $f:[c, d] \rightarrow[c, d]$ is long-branched provided there is some $\delta>0$ such that, for each $n \in \mathbb{N}$, if $[a, b]$ is a maximal interval of monotonicity for $f^{n}$ (a monotonic branch of $f^{n}$ ) then $\left|f^{n}(a)-f^{n}(b)\right| \geq \delta$.

The spaces we consider all have a single endpoint $\overline{0}=(\ldots, 0,0)$.

Definition 2.2 A point $x \in K_{S}$ is called an endpoint if for any two subcontinua $A, B \subset K_{S}$ such that $x \in A \cap B$, we have either $A \subseteq B$ or $B \subseteq A$.

Since $\lim _{\longleftarrow}\left\{[0,1], T_{s}\right\}=\lim _{\longleftarrow}\left\{[0, s / 2],\left.T_{s}\right|_{[0, s / 2]}\right\}$ and $\left.T_{s}\right|_{[0, s / 2]}$ is surjection, from now on, we will work with the restrictions $\left.T_{s}\right|_{[0, s / 2]}$. Note that $\left.T_{S}\right|_{\left[T_{s}(s / 2), s / 2\right]}$ is locally eventually onto:

Definition 2.3 Let $f:[c, d] \rightarrow[c, d]$. We say that $f$ is locally eventually onto (or l.e.o.) provided that for every open set $U \subseteq[c, d]$ there is some integer $n$ such that $f^{n}(U)=[c, d]$.

The interval $\left[T_{s}(s / 2), s / 2\right]$ is called the core of the map $T_{s}$. Notice also that for each $0<\epsilon<s / 2$ there exists an integer $n$ such that $T_{s}^{n}(\epsilon, s / 2]=\left[T_{s}(s / 2), s / 2\right]$.

Now we recall a symbolic representation of the inverse limit space $K_{s}$ provided by Brucks and Diamond in [12]. Since we will work with several types of sequences, to avoid confusion, we denote:

- left-infinite sequences by $\overleftarrow{x}=\left(x_{-i}\right)_{i \in \mathbb{N}}=\cdots x_{-3} x_{-2} x_{-1}$,

- right-infinite sequences by $\vec{x}=\left(x_{i}\right)_{i \in \mathbb{Z}_{+}}=x_{0} x_{1} x_{2} \cdots$,

- bi-infinite sequences by $\bar{x}=\left(x_{i}\right)_{i \in \mathbb{Z}}=\cdots x_{-2} x_{-1} x_{0} x_{1} x_{2} \cdots$.

If $A=a_{0} \cdots a_{k}$ is a finite sequence, $\vec{x}=\left(x_{i}\right)_{i \in \mathbb{Z}_{+}}$and $\overleftarrow{y}=\left(y_{-i}\right)_{i \in \mathbb{N}}$, then

$$
\begin{aligned}
& A^{n \vec{x}}=\underbrace{a_{0} \cdots a_{k} \cdots a_{0} \cdots a_{k}}_{n \text { times }} x_{0} x_{1} \cdots, \\
& \overleftarrow{y} A^{n}=\cdots y_{-2} y_{-1} \underbrace{a_{0} \cdots a_{k} \cdots a_{0} \cdots a_{k}}_{n \text { times }}
\end{aligned}
$$

and $\overleftarrow{y} \vec{x}=\cdots y_{-2} y_{-1} x_{0} x_{1} \cdots$

Definition 2.4 For every point $\xi \in[0, s / 2]$ an itinerary of $\xi$ under the map $T_{s}$ is a right-infinite sequences of zeros and ones $\vec{x}(\xi)=\vec{x}=\left(x_{i}\right)_{i \in \mathbb{Z}_{+}} \in\{0,1\}^{\mathbb{Z}_{+}}$, where

$$
x_{i}= \begin{cases}0, & T_{s}^{i}(\xi) \leq 1 / 2 ; \\ 1, & T_{s}^{i}(\xi) \geq 1 / 2 .\end{cases}
$$


Note that every point $\xi \in[0,1]$ has at most two itineraries and the points which have two itineraries are the preimages of the critical point. The kneading sequence of the map $T_{s}$, denoted by $\vec{c}_{1}=\left(c_{i}\right)_{i \in \mathbb{N}}$, is the itinerary of $T_{s}(1 / 2)=s / 2$. Note that for $i \in \mathbb{N}, \vec{c}_{i}=c_{i} c_{i+1} c_{i+2} \cdots$ is the itinerary of $T_{s}^{i}(1 / 2)$ and that the itinerary of the point 0 is $\overrightarrow{0}^{\infty}=000 \cdots$.

Since $1 / 2$ is a nonrecurrent critical point, there exists a number $R$ such that $c_{1} \cdots c_{R} \neq$ $c_{i+1} \cdots c_{i+R}$, for every $i \in \mathbb{N}$, and such that $R$ is minimal with respect to this property.

Definition 2.5 A sequence $\vec{x} \in\{0,1\}^{\mathbb{Z}_{+}}$is called allowed (with respect to $T_{S}$ ) if there is some point $\xi \in[0,1]$ such that $\vec{x}$ is the itinerary of $\xi$ under the map $T_{s}$.

By Theorem II.3.8 in [16], $\vec{x}$ is allowed if and only if $\overrightarrow{0}^{\infty} \preceq \vec{x}$ and $\sigma^{k} \vec{x} \preceq \vec{c}_{1}$, for every $k \in \mathbb{Z}_{+}$, where $\sigma$ is the one-sided shift ie, $\sigma\left(\left(x_{i}\right)_{i \in \mathbb{Z}_{+}}\right)=\left(x_{i+1}\right)_{i \in \mathbb{Z}_{+}}$, and $\preceq$ is the parity-lexicographic ordering on sequences. Let $X_{S}^{+}$be the set of all allowed sequences $\vec{x} \in\{0,1\}^{\mathbb{Z}_{+}}$,

$$
X_{s}^{+}=\left\{\vec{x} \in\{0,1\}^{\mathbb{Z}_{+}}: \vec{x} \text { is allowed }\right\} .
$$

The metric $d$ on the space $X_{s}^{+}$is given as follows: For two sequences $\vec{x}=\left(x_{i}\right)_{i \in \mathbb{Z}_{+}}$ and $\vec{y}=\left(y_{i}\right)_{i \in \mathbb{Z}_{+}}$, let

$$
d(\vec{x}, \vec{y})= \begin{cases}0, & \text { if } \vec{x}=\vec{y} \\ 2^{-k}, & \text { if } k=\min \left\{j \in \mathbb{Z}_{+}: x_{j} \neq y_{j}\right\} .\end{cases}
$$

The one-sided shift $\sigma: X_{s}^{+} \rightarrow X_{s}^{+}$is continuous with respect to this metric. Define an equivalence relation $\sim$ on $X_{s}^{+}$as follows:

$$
\vec{x} \sim \vec{y}
$$

if either

(1) $\vec{x}=\vec{y}$ or

(2) there exists $m \in \mathbb{Z}_{+}$, such that

(a) $x_{0} x_{1} \cdots x_{m-1}=y_{0} y_{1} \cdots y_{m-1}$,

(b) $x_{m} \neq y_{m}$ and

(c) $\vec{x}_{m+1}=x_{m+1} x_{m+2} \cdots=y_{m+1} y_{m+2} \cdots=\vec{y}_{m+1}=\vec{c}_{1}$.

Let $[\vec{x}]$ denote the equivalence class of $\vec{x}$ in the quotient space $X_{s}^{+} / \sim$. If $[\vec{x}] \in$ $X_{s}^{+} / \sim$ and there exists $\vec{y} \in[\vec{x}]$ with $\vec{y} \neq \vec{x}$, we will write, for simplicity,

$$
[\vec{x}]=x_{0} x_{1} \cdots x_{m-1} \frac{0}{1} \vec{c}_{1} \text {. }
$$


The mapping $\pi: X_{s}^{+} / \sim \rightarrow[0, s / 2]$, given by $\pi[\vec{x}]=\xi$ if $\vec{x}$ is an itinerary of the point $\xi$, is a homeomorphism, and $\pi(\widetilde{\sigma}([\vec{x}]))=T_{s}(\pi([\vec{x}]))$, for every $[\vec{x}] \in X_{s}^{+} / \sim$, where $\tilde{\sigma}: X_{s}^{+} / \sim \rightarrow X_{S}^{+} / \sim$ is given by $\tilde{\sigma}([\vec{x}])=[\sigma \vec{x}]$. For this reason, we will often identify $[0, s / 2]$ and $X_{s}^{+} / \sim$.

For a bi-infinite sequence $\bar{x}=\left(x_{i}\right)_{i \in \mathbb{Z}}$, we denote the right-infinite sequence (also called a right tail of $\bar{x}) x_{j} x_{j+1} x_{j+2} \cdots$ by

$$
\vec{x}_{j}=x_{j} x_{j+1} x_{j+2} \cdots \text {. }
$$

Definition 2.6 A bi-infinite sequence $\bar{x} \in\{0,1\}^{\mathbb{Z}}$ is called allowed (with respect to $T_{S}$ ), if all of its right tails $\vec{x}_{j}$ are itineraries (with respect to $T_{S}$ ).

This is equivalent to assuming that for every right tail $\vec{x}_{j}, j \in \mathbb{Z}$, we have $\overrightarrow{0}^{\infty} \preceq$ $\vec{x}_{j}$ and $\sigma^{k} \vec{x}_{j} \preceq \vec{c}_{1}$, for every $k \in \mathbb{Z}_{+}$. Let

$$
X_{S}=\left\{\bar{x} \in\{0,1\}^{\mathbb{Z}}: \bar{x} \text { is allowed with respect to } T_{s}\right\}
$$

denote the space of all bi-infinite allowed sequences with respect to $T_{S}$.

The metric $d$ on the space $X_{s}$ is given as follows: Let $\bar{x}, \bar{y} \in X_{s}, \bar{x}=\left(x_{i}\right)_{i \in \mathbb{Z}}$, $\bar{y}=\left(y_{i}\right)_{i \in \mathbb{Z}}$. If $\bar{x} \neq \bar{y}$, let $k=\min \left\{|j|: j \in \mathbb{Z}, x_{j} \neq y_{j}\right\}$. Then

$$
d(\bar{x}, \bar{y})= \begin{cases}0, & \text { if } \bar{x}=\bar{y} \\ 2^{-k}, & \text { if } \bar{x} \neq \bar{y}\end{cases}
$$

The shift map $\sigma: X_{S} \rightarrow X_{S}$ given by

$$
(\sigma \bar{x})_{i}=x_{i+1},
$$

for every $i \in \mathbb{Z}$, is a homeomorphism. Define an equivalence relation $\approx$ on the space $X_{s}$ as follows: Two sequences $\bar{x}, \bar{y} \in X_{s}, \bar{x}=\left(x_{i}\right)_{i \in \mathbb{Z}}, \bar{y}=\left(y_{i}\right)_{i \in \mathbb{Z}}$, are equivalent,

$$
\bar{x} \approx \bar{y}
$$

if either

(1) $\bar{x}=\bar{y}$, or

(2) if there is $k \in \mathbb{Z}$ with

(a) $x_{i}=y_{i}$, for $i<k$,

(b) $x_{k} \neq y_{k}$ and

(c) $\vec{x}_{k+1}=\vec{y}_{k+1}=\vec{c}_{1}$. 
By [12, Theorem 2.5] there is a homeomorphism $h: X_{S} / \approx \rightarrow K_{S}$ such that $h(\widetilde{\sigma}([\bar{x}]))=$ $\widehat{T}_{S}(h([\bar{x}]))$, for every $[\bar{x}] \in X_{S} / \approx$, where $\tilde{\sigma}: X_{S} / \approx \rightarrow X_{S} / \approx$ is given by

$$
\tilde{\sigma}([\bar{x}])=[\sigma \bar{x}]
$$

and $\widehat{T}_{s}: K_{S} \rightarrow K_{s}$ is given by

$$
\widehat{T}_{S}\left(\ldots, \xi_{-3}, \xi_{-2}, \xi_{-1}\right)=\left(\ldots, \xi_{-2}, \xi_{-1}, T_{S}\left(\xi_{-1}\right)\right),
$$

ie, the maps $\widetilde{\sigma}$ and $\widehat{T}_{s}$ are conjugate. Note that the maps $\widetilde{\sigma}$ and $\hat{T}_{s}$ are homeomorphisms. We will often identify $K_{S}$ and $X_{S} / \approx$. If there is a sequence $\bar{y} \in[\bar{x}]$ with $\bar{y} \neq \bar{x}$, it is unique, and we denote it by $\bar{x}^{*}=\left(x_{i}^{*}\right)_{i \in \mathbb{Z}}$. If there is no such $\bar{y} \in[\bar{x}]$ with $\bar{y} \neq \bar{x}$, we put $\bar{x}^{*}=\bar{x}$. Let $\pi_{j}: X_{s} / \approx \rightarrow[0, s / 2], j \in \mathbb{Z}_{+}$, be the projection on the $j$-th coordinate, ie,

$$
\pi_{j}[\bar{x}]=\pi\left(\vec{x}_{-j}\right)
$$

where $\pi\left(\vec{x}_{-j}\right)=\xi$ if $\vec{x}_{-j}$ is an itinerary of the point $\xi$.

For a bi-infinite sequence $\bar{x}=\left(x_{i}\right)_{i \in \mathbb{Z}}$, we denote the left-infinite sequence (also called the left tail of $\bar{x}) \cdots x_{j-2} x_{j-1} x_{j}$ by

$$
\overleftarrow{x_{j}}=\cdots x_{j-2} x_{j-1} x_{j}
$$

Definition 2.7 A left-infinite sequence $\overleftarrow{x}=\left(x_{-i}\right)_{i \in \mathbb{N}}$ is allowed if for every $k \in \mathbb{N}$ there exists an itinerary, such that its initial segment of length $k$ is the finite sequence $x_{-k} \cdots x_{-1}$.

Note that if $\bar{x}$ is allowed, then all of its left tails $\overleftarrow{x}_{j}$ are allowed. Each left-infinite allowed sequence $\overleftarrow{x}=\cdots x_{-3} x_{-2} x_{-1}$ corresponds to an arc, but we can also say that it describes one arc component in $K_{S}$ since two sequences $\overleftarrow{x}$ and $\overleftarrow{y}$ describe the same arc component if and only if they have a common left tail [12, Corollary 2.10]. So, the arc component described by $\overleftarrow{x}$ is the set of allowed bi-infinite sequences whose left tail eventually coincides with $\overleftarrow{x}$

Let $\overleftarrow{a}=\cdots a_{-3} a_{-2} a_{-1}$ be allowed and let $n \in \mathbb{Z}_{+}$. The set

$$
A_{\overleftarrow{a}}^{n}=\left\{[\bar{x}] \in K_{s}: \exists \bar{x} \in[\bar{x}], \overleftarrow{x}_{-n}=\overleftarrow{a}\right\}
$$

is an arc, and we call it a basic arc.

Let $\overleftarrow{y}$ be a fixed left-infinite allowed sequence, $\overleftarrow{y}=\cdots y_{-3} y_{-2} y_{-1}$, and let $C^{\prime}$ be the corresponding arc component in $K_{s}$. Let $A_{\overleftarrow{v}}^{n}$ be some basic arc contained in $C^{\prime}$. 
Then either

(1) $\overleftarrow{v}_{-1}=\overleftarrow{y}_{-n}$ or

(2) there is some $k \in \mathbb{N}$ with

(a) $v_{-k} \neq y_{-n-k+1}$ and

(b) $\overleftarrow{v}_{-k-1}=\overleftarrow{y}_{-n-k}$

In the first case we write only $A^{n}$ instead of $A_{\overleftarrow{v}}^{n}$ or $A_{\overleftarrow{y}_{-n}}^{n}$. In the second case, $k>0$, we write only $A_{v}^{n}$ instead of $A_{\overleftarrow{v}}^{n}$, where we take $v$ to be the initial segment of $\overleftarrow{v}$ that disagrees with $\overleftarrow{y}$, ie, $v=v_{-k} \cdots v_{-1}$, and we have that $\overleftarrow{v}_{-k-1}=\overleftarrow{y}_{-n-k}$

Before we can describe an ordering on the arc component $C^{\prime}$ that has as its representative the left-infinite allowed sequence $\overleftarrow{y}$, we must first give an indication as to the "orientation" of the basic arcs in $C^{\prime}$. It will be evident that this orientation on $C^{\prime}$ is dependent upon $\overleftarrow{y}$, and there are many possible choices for $\overleftarrow{y}$. However, once a representative left-infinite allowed sequence has been chosen for an arc component, we can define an orientation. To begin, for $n \in \mathbb{N}$, let

$$
P(n)=\operatorname{card}\left\{i: y_{-i}=1,1 \leq i \leq n\right\} .
$$

If $n=0$, let $P(0)=0$. A basic arc

$$
A^{n}=\left\{[\bar{x}] \in K_{s}: \exists \bar{x} \in[\bar{x}], \overleftarrow{x}_{-n}=\overleftarrow{y}_{-n}\right\}
$$

is called even if $P(n)$ is even and it is called $o d d$ if $P(n)$ is odd. An arc $A_{v}^{n}$, where $v=v_{-k} \cdots v_{-1}, v_{-k} \neq y_{-n-k}$, is called even if

$$
(-1)^{P(n+k)}=\prod_{i=1}^{k}(-1)^{v_{-i}}
$$

and it is called odd if

$$
(-1)^{P(n+k)} \neq \prod_{i=1}^{k}(-1)^{v_{-i}} .
$$

In this case the parity of the arc depends on the initial segment of the left-infinite sequence $\overleftarrow{v}$ that disagrees with $\overleftarrow{y}$ and determines $A_{v}^{n}$

Note that if two basic arcs $A_{\overleftarrow{v}}^{n}$ and $A_{\overleftarrow{u}}^{n}$ are adjacent, ie, if two basic arcs $A_{\overleftarrow{v}}^{n}$ and $A_{\overleftarrow{u}}^{n}$ have a common boundary point, then $\overleftarrow{v}$ and $\overleftarrow{u}$ disagree in only one coordinate Therefore, for any pair of adjacent basic arcs of the arc component $C^{\prime}$, one of them is even and the other one is odd with respect to the previously defined orientation. On the other hand, if $[\bar{x}],[\bar{y}]$ are boundary points of $A_{\overleftarrow{v}}^{n}$ and $[\bar{y}],[\bar{z}]$ are boundary 
points of $A_{\overleftarrow{u}}^{n}$, then $\vec{x}_{-n} \prec \vec{y}_{-n}$ if and only if $\vec{z}_{-n} \prec \vec{y}_{-n}$ (by $\prec$ we mean the paritylexicographic ordering on sequences).

We now introduce an ordering on the arc component $C^{\prime}$ with representative $\overleftarrow{y}$ denoted by $\preceq$ and called generalized parity-lexicographical ordering, as follows: For $[\bar{x}],[\bar{z}] \in$ $C^{\prime}$, let

$$
k=k([\bar{x}],[\bar{z}])=\max \left\{i \in \mathbb{N}: x_{-i} \neq y_{-i} \text { or } z_{-i} \neq y_{-i}\right\} .
$$

If $x_{-i}=y_{-i}$ and $z_{-i}=y_{-i}$, for all $i \in \mathbb{N}, \bar{x} \in[\bar{x}], \bar{z} \in[\bar{z}]$, let $k=0$. We say that $\bar{x} \prec \bar{z}$ if either

(1) $(-1)^{P(k)} x_{-k}<(-1)^{P(k)} z_{-k}$ or

(2) there exists $l>-k$, such that

(a) $x_{i}=z_{i}$, for $-k \leq i<l$ and

(b) $(-1)^{P(k)} \varepsilon x_{l}<(-1)^{P(k)} \varepsilon z_{l}$, where

$$
\varepsilon=\prod_{i=-k}^{l-1}(-1)^{x_{i}}=\prod_{i=-k}^{l-1}(-1)^{z_{i}} \in\{-1,1\} \text {. }
$$

We say that $[\bar{x}] \preceq[\bar{z}]$ if $\bar{x} \prec \bar{z}$ or $\bar{x}=\bar{z}$.

Let us explain the geometrical meaning of the above formula in an example.

Example For simplicity, let us consider the composant $C$ containing the endpoint [0 $]$. Let $C$ be described by the left tail $\overleftarrow{0}^{\infty}$. Since every kneading sequence starts with 10 and since $00 \prec 01 \prec 11 \prec 10$, the following holds: The left tail $\overleftarrow{0}^{\infty}$ represents the basic $\operatorname{arc} A^{1}=A_{\ldots 00}^{1}$ containing the endpoint $[\overline{0}]$. The adjacent basic arc $A_{1}^{1}=A_{\ldots 001}^{1}$ is represented by the left tail $\overleftarrow{0}^{\infty} 1$. The next basic arc is $A_{\ldots 0011}^{1}=A_{11}^{1}$ represented by the left tail $\overleftarrow{0}^{\infty} 11$, and the fourth basic arc $A_{\ldots 0010}^{1}=A_{10}^{1}$ is represented by $\overleftarrow{0}^{\infty} 10$; see Figure 1.

The basic arc $A^{1}$ is even since we chose the left tail which represents $A^{1}$ as a representative of the composant. Also, since the composant is described by $\overleftarrow{0^{\infty}}$, then $P(n)=0$ for every $n \in \mathbb{N}$. The basic arc $A_{1}^{1}$ is odd since $P(1)=0$ and $(-1)^{0} \neq(-1)^{1}$. The $\operatorname{arc} A_{11}^{1}$ is even since $P(2)=0$ and $(-1)^{0}=(-1)^{1}(-1)^{1}$. The arc $A_{10}^{1}$ is odd since $P(2)=0$ and $(-1)^{0} \neq(-1)^{0}(-1)^{1}$.

Let $\left[\bar{x}^{1}\right],\left[\bar{x}^{2}\right],\left[\bar{x}^{3}\right] \in C$ be chosen such that $A^{1} \cap A_{1}^{1}=\left\{\left[\bar{x}^{1}\right]\right\}, A_{1}^{1} \cap A_{11}^{1}=\left\{\left[\bar{x}^{2}\right]\right\}$ and $A_{11}^{1} \cap A_{10}^{1}=\left\{\left[\bar{x}^{3}\right]\right\}$; see Figure 1. According to the definition of the generalized paritylexicographical ordering, we have $[\overline{0}] \prec\left[\bar{x}^{1}\right]$ because $A^{1}$ is even and $\overrightarrow{0}_{-1} \prec \vec{x}_{-1}^{1}$. Moreover, for every pair of points $[\bar{y}],[\bar{z}] \in A^{1}$, if $\vec{y}_{-1} \prec \vec{z}_{-1}$ then $[\bar{y}] \prec[\bar{z}]$. 


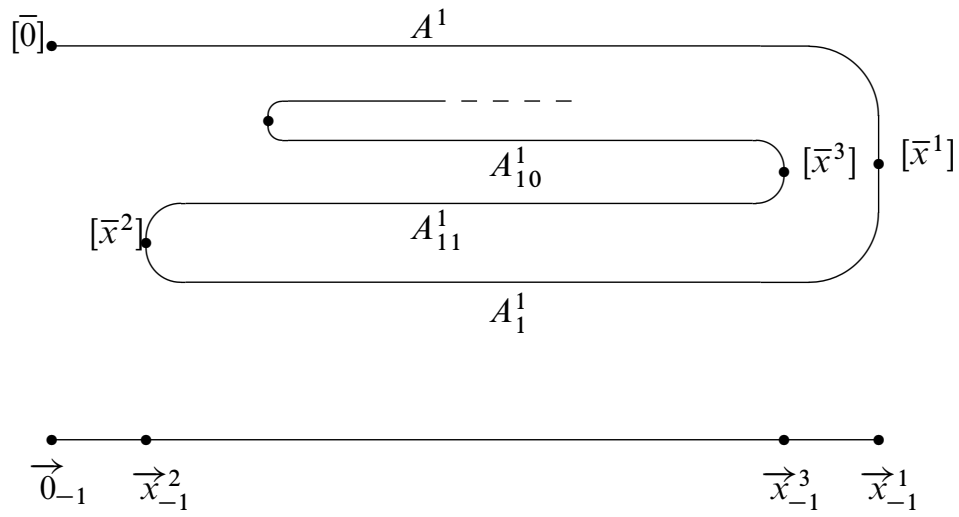

Figure 1: Example

Next notice that $\left[\bar{x}^{1}\right] \prec\left[\bar{x}^{2}\right]$ even though $\vec{x}_{-1}^{2} \prec \vec{x}_{-1}^{1}$ because the basic arc containing them, $A_{1}^{1}$, is odd. It is also the case that for every pair of points $[\bar{y}],[\bar{z}] \in A_{1}^{1}$ if $\vec{y}_{-1} \prec \vec{z}_{-1}$ then $[\bar{z}] \prec[\bar{y}]$ again because this arc is odd.

Proceeding, we see that for any two points $[\bar{y}],[\bar{z}] \in C$ there is an $n \in \mathbb{N}$ and a finite word $v$ such that $[\bar{y}],[\bar{z}] \in A_{v}^{n}$, a basic arc. As above, if $A_{v}^{n}$ is even and $\vec{y}_{-n} \prec \vec{z}_{-n}$ then $[\bar{y}] \prec[\bar{z}]$. If instead though $A_{v}^{n}$ is odd and $\vec{y}_{-n} \prec \vec{z}_{-n}$ then $[\bar{z}] \prec[\bar{y}]$.

If $C^{\prime}$ is an arc component which does not contain the endpoint, there exists an orderpreserving bijection $\phi$ between the real line, endowed with its natural order, and $C^{\prime}$, endowed with the ordering $\preceq$. For the composant $C$ containing the endpoint $[\overline{0}]$, there exists an order-preserving bijection $\varphi$ between the half line, endowed with its natural order, and $C$, endowed with the ordering $\preceq$. Therefore, the ordering $\preceq$ is natural. Note that $\phi$ and $\varphi$ are continuous, the inverse of $\phi$ is not continuous, whereas the inverse of $\varphi$ is continuous.

In this paper we focus on the distinguished composant containing the unique endpoint $[\overline{0}]=[\cdots 00.00 \cdots]$, and from now on let $C$ be the composant containing the point $[\overline{0}]$. We define some special points as follows:

Definition 2.8 A point $[\bar{x}] \in C$ is called an identification point if there is an $m \in \mathbb{Z}_{+}$ with $\vec{x}_{-m+1}=\vec{c}_{1}$. The level of an identification point $[\bar{x}]$ is defined by $L[\bar{x}]=m$ if $x_{-m} \neq x_{-m}^{*}$. That is to say $\pi_{m}([\bar{x}])=\pi\left(\vec{x}_{-m}\right)=1 / 2$.

The importance of the identification points and their levels can be seen by considering the following: Let $\overleftarrow{a}=\left(a_{-i}\right)_{i \in \mathbb{N}}$ and $\stackrel{b}{b}=\left(b_{-i}\right)_{i \in \mathbb{N}}, \overleftarrow{a} \neq \overleftarrow{b}$, be allowed sequences. For 
$n \in \mathbb{N}$, let $A_{\overleftarrow{a}}^{n}$ and $A_{\overleftarrow{b}}^{n}$ be the associated basic arcs. If there is a point $[\bar{x}] \in A_{\overleftarrow{a}}^{n} \cap A_{\overleftarrow{b}}^{n}$, then

$$
\overleftarrow{x}_{-n}=\overleftarrow{a} \text { and } \overleftarrow{x}_{-n}^{*}=\overleftarrow{b}
$$

Hence, $[\bar{x}]$ is an identification point, and there is $m \geq n$ with

$$
\begin{gathered}
\overleftarrow{x}_{-m-1}=\overleftarrow{x}_{-m-1}^{*}=\overleftarrow{a}_{n-m-2}, \\
x_{-m} \neq x_{-m}^{*} \text { and } \vec{x}_{-m+1}=\vec{x}_{-m+1}^{*}=\vec{c}_{1}
\end{gathered}
$$

implying that $L[\bar{x}]=m$. Also, if $[\bar{y}] \in A_{\overleftarrow{a}}^{n}$ is an identification point with $L[\bar{y}]>n$, then $[\bar{y}] \in \partial A_{\overleftarrow{a}}^{n}$.

Note that the restriction of $\pi_{i}$ to $A_{\overleftarrow{a}}^{n}$ is an injection, for all $i \geq n-1$, and if $A_{\overleftarrow{a}}^{n}$ has boundary points $[\bar{x}]$ and $[\bar{y}]$ with $L[\bar{x}]=l$ and $L[\bar{y}]=k$, then

$$
\pi_{n-1}\left(A_{\overleftarrow{a}}^{n}\right)=\left\{\pi_{n-1}[\bar{x}]:[\bar{x}] \in A_{\overleftarrow{a}}^{n}\right\}
$$

is a closed interval with boundary points $T_{s}^{l-n+1}(1 / 2)$ and $T_{s}^{k-n+1}(1 / 2)$. Let $A_{\overleftarrow{b}}^{n}$ be another basic arc. Let $\left\{\left[\bar{x}^{0}\right] \prec \cdots \prec\left[\bar{x}^{i}\right]\right\}$ be the ordered set of all identification points of $A_{\overleftarrow{a}}^{n}$, and $\left\{\left[\bar{u}^{0}\right] \prec \cdots \prec\left[\bar{u}^{j}\right]\right\}$ be the ordered set of all identification points of $A_{\overleftarrow{b}}^{n}$. If $\pi_{n-1}\left(\partial A_{\overleftarrow{a}}^{n}\right)=\pi_{n-1}\left(\partial A_{\overleftarrow{b}}^{n}\right)$, then $i=j$ and either

(1) $L\left[\bar{x}^{m}\right]=L\left[\bar{u}^{m}\right]$, for every $m \in\{0, \ldots, j\}$, if $A_{\overleftarrow{a}}^{n}$ and $A_{\overleftarrow{b}}^{n}$ have the same parity or

(2) $L\left[\bar{x}^{m}\right]=L\left[\bar{u}^{j-m}\right]$, for every $m \in\{0, \ldots, j\}$, if they have different parity.

For every $k \in\{0, \ldots, n-1\}$, the $\operatorname{arc} A_{\overleftarrow{a}}^{n}$ is a union of $\operatorname{arcs} A_{w}^{k}$, ie,

$$
A_{\overleftarrow{a}}^{n}=\bigcup_{w} A_{w}^{k}
$$

where the union is computed over all finite sequences $w$ of length $n-k$ such that $\overleftarrow{a} w$ is allowed. Since $\left.T_{s}\right|_{\left[T_{s}(s / 2), s / 2\right]}$ is 1.e.o. and $\pi \circ \sigma=T_{s} \circ \pi$ for every arc $A$, there is an $m \in \mathbb{Z}_{+}$such that

$$
\widetilde{\sigma}^{m}(A)=\left\{\widetilde{\sigma}^{m}[\bar{x}]:[\bar{x}] \in A\right\}
$$

contains at least one identification point.

We stratify the collection of identification points into the following subcollections:

Definition 2.9 For every $p \in \mathbb{Z}_{+}$a point $[\bar{x}] \in C$ is called a $p$-point, if either there is an $m \in \mathbb{Z}_{+}$with $\left[\vec{x}_{-p-m+1}\right]=\left[\vec{c}_{1}\right]$ or if $[\bar{x}]=[\overline{0}]$. A $p$-point $[\bar{x}]$ has $p$-level $L_{p}[\bar{x}]=m$ if $x_{-p-m} \neq x_{-p-m}^{*}$. Define $L_{p}[\overline{0}]=\infty$, for every $p \in \mathbb{Z}_{+}$. 
For every $p, m \in \mathbb{Z}_{+}$, the set

$$
E_{p, m}=\left\{[\bar{x}] \in C: \exists \bar{x} \in[\bar{x}], \vec{x}_{-p-m+1}=\vec{c}_{1}\right\}
$$

is the set of all $p$-points of level $m$ and

$$
E_{p}=\bigcup_{m=0}^{\infty} E_{p, m} \cup\{[\overline{0}]\}
$$

is the set of all $p$-points of the composant $C$. Note that $E_{p+1} \subset E_{p}$ for every $p \in \mathbb{Z}_{+}$. Since there is an order-preserving bijection from $\left(\mathbb{Z}_{+}, \leq\right)$to $\left(E_{p}, \preceq\right)$, such that $0 \in \mathbb{Z}_{+}$ is mapped to $[\overline{0}] \in E_{p}$, from now on, the points of $E_{p}$ will be indexed by $\mathbb{Z}_{+}$. So, $E_{p}=\left\{\left[\bar{x}^{0}\right],\left[\bar{x}^{1}\right],\left[\bar{x}^{2}\right], \ldots\right\}$ and $\left[\bar{x}^{0}\right]=[\overline{0}]$.

Definition 2.10 The sequence of integers $L_{p}\left[\bar{x}^{0}\right], L_{p}\left[\bar{x}^{1}\right], L_{p}\left[\bar{x}^{2}\right], \ldots$ is called the folding pattern of the composant $C$.

Let $q \in \mathbb{Z}_{+}, q>p$, and $E_{q}=\left\{\left[\bar{y}^{0}\right],\left[\bar{y}^{1}\right],\left[\bar{y}^{2}\right], \ldots\right\}$. Since $\widetilde{\sigma}^{q-p}$ is an order-preserving homeomorphism on $C$, it is easy to see that, for every $i \in \mathbb{Z}_{+}, \widetilde{\sigma}^{q-p}\left(\left[\bar{x}^{i}\right]\right)=\left[\bar{y}^{i}\right]$ and $L_{p}\left[\bar{x}^{i}\right]=L_{q}\left[\bar{y}^{i}\right]$. Therefore, the folding pattern of the composant $C$ does not depend on $p$.

Note that the arc between any two adjacent $p$-points $\left[\bar{x}^{j}\right],\left[\bar{x}^{j+1}\right]$ is a basic arc $A_{\overleftarrow{y}-p}^{p}$, where $\overleftarrow{y}_{-p}$ is a left tail of any point $[\bar{y}]$ between $\left[\bar{x}^{j}\right],\left[\bar{x}^{j+1}\right]$. Also, for every $[\bar{x}]^{y-p} \in \operatorname{Int} A_{\overleftarrow{y}_{-p}}^{p}$ either $x_{-p}=0$, or $x_{-p}=1$

Definition 2.11 Let $A_{\overleftarrow{y}}^{p}$ be a basic arc. We say that $A_{\overleftarrow{y}}^{p}$ is a basic arc of sign 0 (respectively of sign 1), if $x_{-p}=0$ (respectively $\left.x_{-p}=1\right)$ ), for every $[\bar{x}] \in \operatorname{Int} A_{\overleftarrow{y}}^{p}$.

Remark 2.12 If $A_{\overleftarrow{x}}^{p}$ and $A_{\overleftarrow{y}}^{p}$ are two adjacent basic arcs and $[\bar{z}] \in A_{\overleftarrow{x} p}^{p} \cap A_{\overleftarrow{y}}^{p}$, then $A_{\overleftarrow{x}}^{p}$ and $A_{\overleftarrow{y}}^{p}$ have the same sign if and only if $L_{p}[\bar{z}]>0$. That is, $A_{\overleftarrow{x}}^{p}$ and $A_{\overleftarrow{y}}^{p}$ have different signs if and only if $L_{p}[\bar{z}]=0$.

Let $A$ be an arc of the composant $C$ such that $\partial A=\{[\bar{u}],[\bar{v}]\}$ and $A \cap E_{p}=$ $\left\{\left[\bar{y}^{0}\right], \ldots,\left[\bar{y}^{n}\right]\right\}$. Let us assume that if a point from $\partial A$ is a $p$-point, then its $p-$ level is greater than zero. Then the first paragraph of this remark implies $u_{-p}=v_{-p}$ if and only if the number of $p$-points in $A$ with zero $p$-level is even, ie, $\operatorname{card}\left\{\left[\bar{y}^{i}\right]\right.$ : $\left.L_{p}\left[\bar{y}^{i}\right]=0,0 \leq i \leq n\right\}$ is even. Similarly, if every $p$-point from $\partial A$ has $p$-level which is not equal to $l$, then $u_{-p-l}=v_{-p-l}$ if and only if the number of $p$-points in $A$ with $p$-level equal to $l$ is even, ie, $\operatorname{card}\left\{\left[\bar{y}^{i}\right]: L_{p}\left[\bar{y}^{i}\right]=l, 0 \leq i \leq n\right\}$ is even.

In Figure 2 we have $n=4$. Recall that every $p$-point $\left[\bar{y}^{i}\right]$ is "coded" by a pair of biinfinite sequences which agree in all coordinates except one. In the figure we represent 
every such pair by a horizontal line, where we emphasize some coordinates of particular interest for this example by writing them explicitly. If the particular coordinate is the coordinate where two bi-infinite sequences disagree, we write double coordinate as $\frac{0}{1}$ or $\frac{1}{0}$.

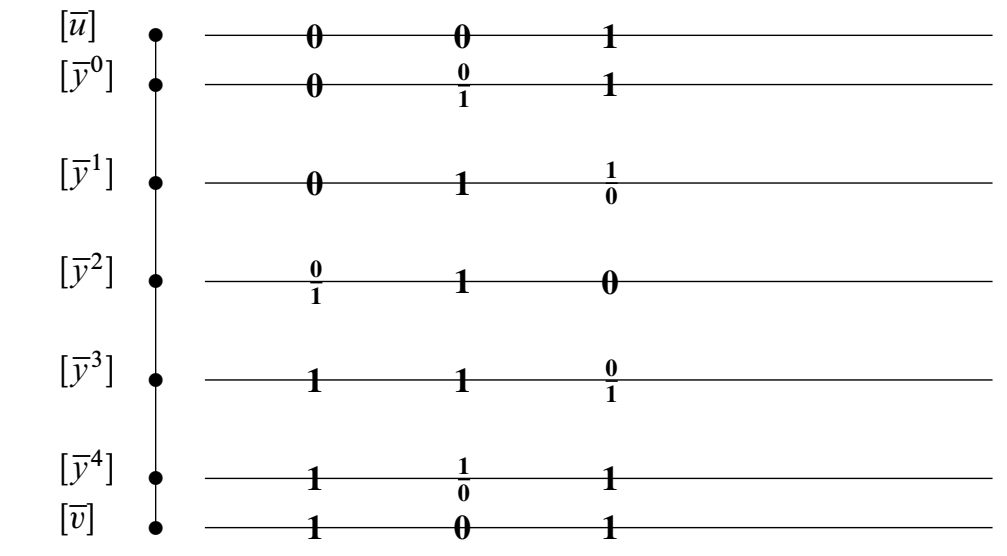

Coordinate: $\quad-p-2 \quad-p-1 \quad-p$

Figure 2: Example for Remark 2.12.

Let $A$ be an arc of the composant $C$ and $A \cap E_{p}=\left\{\left[\bar{y}^{0}\right], \ldots,\left[\bar{y}^{n}\right]\right\}$. We call the finite sequence

$$
\mathrm{FP}_{p}(A)=L_{p}\left[\bar{y}^{0}\right], \ldots, L_{p}\left[\bar{y}^{n}\right]
$$

the $p$-folding pattern of the arc $A$. We will write, for simplicity, $\operatorname{FP}(A)$ instead of $\mathrm{FP}_{p}(A)$, whenever it causes no confusion.

We now recall some definitions and properties introduced and proven in [26].

Definition 2.13 An arc $A$ of the composant $C$ such that $\partial A=\{[\bar{u}],[\bar{v}]\}$ and $A \cap E_{p}=$ $\left\{\left[\bar{y}^{0}\right], \ldots,\left[\bar{y}^{n}\right]\right\}$ is called $p$-symmetric if

$$
\left[\vec{u}_{-p}\right]=\left[\vec{v}_{-p}\right] \text { and } L_{p}\left[\bar{y}^{i}\right]=L_{p}\left[\bar{y}^{n-i}\right],
$$

for every $0 \leq i \leq n$.

Every $p$-symmetric arc is also $q$-symmetric, for every $0 \leq q \leq p$. If $A \subset C$ is a $p$-symmetric arc, and $A \cap E_{p}=\left\{\left[\bar{x}^{0}\right], \ldots,\left[\bar{x}^{n}\right]\right\}$, then $n$ is even. For the $p$-point $\left[\bar{x}^{n / 2}\right]$, called the center of $A$, and denoted by $\left[\bar{\chi}^{A}\right]$, we have

$$
L_{p}\left[\bar{\chi}^{A}\right]=\max \left\{L_{p}[\bar{x}]:[\bar{x}] \in E_{p} \cap A\right\} .
$$


Definition 2.14 For $p \in \mathbb{Z}_{+}$an arc $B$ of the composant $C$ is called a $p$-bridge if $\partial B \subset E_{p}, L_{p}[\bar{x}]=0$, for every $[\bar{x}] \in \partial B$, and $L_{p}[\bar{x}] \neq 0$, for every $[\bar{x}] \in \operatorname{Int} B \cap E_{p}$.

It is easy to see that $p$-bridges are $p$-symmetric, and that $L_{p}\left[\bar{\chi}^{B}\right]$ determines the $q$-folding pattern of the $p$-bridge $B$, for all $q \leq p$ (see Lemma 3.8 in [29]).

Lemma 2.15 [26, Corollary 3.2] If $c_{3}=0$, then, for every $n \in \mathbb{N}$, there is a $p$-bridge $B \subset C$ such that $L_{p}\left[\bar{\chi}^{B}\right]=n$.

Lemma 2.16 [26, Lemma 3.3] For every $n \in \mathbb{N}$, there is a $p$-bridge $B \subset C$ such that $L_{p}\left[\bar{\chi}^{B}\right]=2 n$.

Lemma 2.17 [26, Lemma 3.4] Let $C^{\prime}$ be a non-endpoint composant of $K_{S}$. Let $m=\min \left\{i \in \mathbb{N}: c_{2 i+1}=0\right\}$. There is a $p$-bridge $B \subset C^{\prime}$ such that $L_{p}\left[\bar{\chi}^{B}\right]=2 n-1$ if, and only if, $n \geq m$.

Remark 2.18 The endpoint composant, in contrast, has a $p$-bridge with centers of every level. However, the situation for Lemma 2.17 is not so different in the case that $B$ is a $p$-bridge in the endpoint composant. Even though the center of $B$ could have any level, in that case there is only one $p$-bridge $B \subset C$ with $L_{p}\left[\bar{\chi}^{B}\right]=2 n-1$ with $n<m$. So there is a point $[\bar{x}] \in C$ such that if $B$ is a $p$-bridge of $C$ that occurs after $[\bar{x}]$, then $L_{p}\left[\bar{\chi}^{B}\right]=2 n-1$ if, and only if, $n \geq m$. We lose no generality in assuming that all of the $p$-bridges in $C$ that we consider occur in $C$ after this point $[\bar{x}]$.

Theorem 2.19 [26, Theorem 3.6] Let $p \in \mathbb{Z}_{+}$. There exists $M \in \mathbb{N}$ such that for every $p$-bridge $B \subset C$ and for every $[\bar{x}] \in B \cap E_{p},[\bar{x}] \neq\left[\bar{\chi}^{B}\right], L_{p}[\bar{x}]<M$.

This means that, in the nonrecurrent case, every point $[\bar{x}] \in E_{p}$ with $L_{p}[\bar{x}] \geq M$ is the center of some $p$-bridge. This also means that the number of $p$-points of a $p$-bridge is bounded, ie, there exists $L \in \mathbb{N}$ such that for a $p$-bridge $B \subset C, \operatorname{card}\left(B \cap E_{p}\right) \leq L$.

Lemma 2.20 [26, Lemma 3.7] Let $p \in \mathbb{Z}_{+}$. Let $B \subset C$ be a $p$-bridge and $B \cap E_{p}=$ $\left\{\left[\bar{x}^{0}\right], \ldots,\left[\bar{x}^{n}\right]\right\}$. Let $A$ be the arc between the points $\left[\bar{x}^{0}\right]$ and $\left[\bar{x}^{1}\right]$. Then we have $\left\{\left[\vec{x}_{-p}\right]:[\bar{x}] \in A\right\}=\left\{\left[\vec{x}_{-p}\right]:[\bar{x}] \in B\right\}$.

Now we recall the definition of a family of chains of $K_{S}$ introduced in [29]. Let $V^{n}$ be the set of all allowed sequences of length $n$ ordered by the parity-lexicographical ordering. This set is not empty and it is finite. Let $k_{n}=\operatorname{card}\left(V^{n}\right)$. 
Definition 2.21 For $m \in \mathbb{Z}_{+}, n \in \mathbb{N}$, let

$$
V^{m+n+1}=\left\{v^{i}: i=0, \ldots, k_{m+n+1}-1\right\} .
$$

For $i \in\left\{1, \ldots, k_{m+n+1}-1\right\}$, let

$$
\ell_{m, n}^{i}=\left\{[\bar{x}] \in K_{s}: \forall \bar{u} \in[\bar{x}], u_{-m} \cdots u_{n} \in\left\{v^{i-1}, v^{i}\right\}\right\} .
$$

Let

$$
\mathcal{C}_{m, n}=\left\{\ell_{m, n}^{i}: i=1, \ldots, k_{m+n+1}-1\right\}
$$

It is easy to see that $\mathcal{C}_{m, n}$ is an open chaining of $K_{s}$.

Let $\mathcal{C}$ and $\mathcal{C}^{\prime}$ be chainings. We write $\mathcal{C}^{\prime} \prec \mathcal{C}$ to mean that $\mathcal{C}^{\prime}$ refines $\mathcal{C}$. Although we use $\prec$ for the ordering on the composant $C$, this will not lead to confusion since from the context it will be clear what category of objects we are comparing.

Lemma 2.22 If $\mathcal{C}$ is an arbitrary chain of open sets of $K_{s}$, then there exist $m \in \mathbb{Z}_{+}$, $n \in \mathbb{N}$, such that $\mathcal{C}_{m, n} \prec \mathcal{C}$. If $m \geq i$ and $n \geq j$, then $\mathcal{C}_{m, n} \prec \mathcal{C}_{i, j}$.

We omit the proof. We call the set

$$
L_{m, n}^{i}=\left\{[\bar{x}] \in K_{s}: \forall \bar{u} \in[\bar{x}], u_{-m} \cdots u_{n}=v^{i}\right\}
$$

the cylinder generated by the word $v^{i}$. We also let $L_{m, n}^{x}$ be the cylinder which contains the point $[\bar{x}]$, and $\ell_{m, n}^{x}$ be a link which contains the point $[\bar{x}]$. Note that if there is a cylinder which contains the point $[\bar{x}]$, it is unique. Whereas for every point there is a link which contains it, but this link is not always unique. There are at most two such links, but for $[\bar{x}] \in E_{p}$ such that $L_{p}[\bar{x}]=l$ with $l \in\{1,2\}$, we can choose $n$ large enough to ensure that the link $\ell_{p, n}^{x}$ is unique (see below).

Remark 2.23 If a $p$-point $[\bar{x}]$ with $L_{p}[\bar{x}]=l$ satisfies $-m \leq-p-l \leq n$, then $[\bar{x}] \notin L_{m, n}^{i}$ for any $i$, but the link $\ell_{m, n}^{x}$ which contains $[\bar{x}]$ is unique. On the other hand, if $-p-l>-m$, then there is a unique cylinder $L_{m, n}^{x}$ which contains $[\bar{x}]$. In this case $L_{m, n}^{x}$ is generated by the word $v^{i}=x_{-m} \cdots x_{n}$. Recall that if $v^{i+1}=v_{0} \cdots v_{m+n}$ is an adjacent word to $v^{i}$, then there is a unique $j, 0 \leq j \leq m+n$, such that $v_{j} \neq x_{-m+j}$. If $j \leq m+n-1$, then also $v_{j+1} \cdots v_{m+n}=x_{-m+j+1} \cdots x_{n}=c_{1} \cdots c_{n+m-j}$. For $-p-l>-m$ we have also $x_{-m} \cdots x_{n}=c_{i} \cdots c_{i+m+m}$ for some $i \in \mathbb{N}$. Since $\vec{c}_{1}$ is not recurrent, then $n+m-j<R$, where $R$ is the smallest natural number such that $c_{1} \cdots c_{R} \neq c_{i+1} \cdots c_{i+R}$, for every $i \in \mathbb{N}$.

Since the critical point $1 / 2$ of $T_{s}$ is not recurrent, there exists an $\epsilon>0$ such that intervals $(1 / 2-\epsilon, 1 / 2+\epsilon),\left(T_{S}(1 / 2)-\epsilon, T_{S}(1 / 2)+\epsilon\right)$ and $\left(T_{S}^{2}(1 / 2)-\epsilon, T_{S}^{2}(1 / 2)+\epsilon\right)$ 
do not contain $T_{s}^{i}(1 / 2)$ for $i>2$. Therefore, there exists some $N \in \mathbb{N}$ such that if $n \geq N$ then the link of $\mathcal{C}_{0, n}$ which contains all 0 -points whose 0 -levels are 1 (respectively 2 ), does not contain 0 -points whose 0 -levels are not 1 (respectively 2 ). In other words, 0 -points which project to $\vec{c}_{1}$ or $\vec{c}_{2}$ in the 0 -th coordinate are not in a link of $\mathcal{C}_{0, n}$ containing 0 -points which project to $\vec{c}_{i}, i>2$, in the 0 -th coordinate. Since $E_{p} \subset E_{0}$ and $\mathcal{C}_{p, n} \prec \mathcal{C}_{0, n}$, for every $p>0$, then also for $n \geq N$, if $[\bar{x}] \in E_{p}$ is such that $L_{p}[\bar{x}]=l$ with $l \in\{1,2\}$, then for every $[\bar{y}] \in E_{p} \cap \ell_{p, n}^{x}, L_{p}[\bar{y}]=l$.

Recall that $T_{s}$ is long-branched, so there is some $\delta>0$ such that for $m \in \mathbb{N}$ if $A=[x, y]$ is a maximal interval of monotonicity for $T_{s}^{m}$ then $\left|T_{s}^{m}(x)-T_{s}^{m}(y)\right|>\delta$. Choose $N \in \mathbb{N}$ large enough so that also if $n \geq N$ then $\operatorname{mesh}\left(\mathcal{C}_{0, n}\right)<\delta$. By $\operatorname{mesh}\left(\mathcal{C}_{0, n}\right)$ we mean the largest diameter of all the links of $\mathcal{C}_{0, n}$. Since $\mathcal{C}_{p, n} \prec \mathcal{C}_{0, n}$, for every $p>0$, then also $\operatorname{mesh}\left(\mathcal{C}_{p, n}\right)<\delta$ for every $p>0$ and $n \geq N$.

\section{Pseudohomotopy construction}

Assume that $s, t \in(\sqrt{2}, 2]$ are such that $T_{s}$ and $T_{t}$ have nonrecurrent, nonpreperiodic critical points, and such that

$$
h: \lim _{\longleftarrow}\left\{[0,1], T_{t}\right\} \rightarrow \underset{\lim }{\longleftarrow}\left\{[0,1], T_{s}\right\}
$$

is a homeomorphism. Let $K_{t}=\lim \left\{[0,1], T_{t}\right\}$ with endpoint composant $C^{\prime}$ and let $K_{s}=\lim \left\{[0,1], T_{s}\right\}$ with endpoint composant $C$. As in the previous section, $T_{t}$ is long-branched, so there is a $\delta^{\prime}>0$ such that if $m \in \mathbb{N}$ and $A^{\prime}$ is a maximal interval of monotonicity for $T_{t}^{m}$ then $\operatorname{diam}\left(T_{t}^{m}(A)\right)>\delta^{\prime}$. Since $1 / 2$ is not recurrent for $T_{t}$, choose $R^{\prime}$ such that the initial segment of $\vec{c}_{1}^{\prime}$ of length $R^{\prime}$ never repeats in $\vec{c}_{1}^{\prime}$.

Let $N^{\prime} \in \mathbb{N}$ be such that $\operatorname{mesh}\left(\mathcal{C}_{q, m}^{\prime}\right)<\delta^{\prime}$ for all $q \in \mathbb{Z}_{+}$and $m \geq N^{\prime}$. Let $N^{\prime}$ be large enough so that also $m \geq N^{\prime}$ implies that $q$-points which have $q$-levels 1 or 2 are not in a link of $\mathcal{C}^{\prime}{ }_{q, m}$ with any $q$-points with different $q$-levels. In a similar manner $N \in \mathbb{Z}_{+}$is defined for $T_{s}, \delta$ and $R$ in the previous section (Remark 2.23). Let $S, S^{\prime} \in \mathbb{N}, S \geq N$ and $S^{\prime} \geq N^{\prime}$ be large enough so that all technical requirements of Remark 4.7 from the Appendix are satisfied.

For this section we assume that $q, p, g \in \mathbb{Z}_{+}, m, e \geq S^{\prime}$ and $n \geq S$ are such that

$$
h\left(\mathcal{C}_{q, m}^{\prime}\right) \preceq \mathcal{C}_{p, n} \preceq h\left(\mathcal{C}_{g, e}^{\prime}\right) .
$$

Lemma 3.1 If $A$ is an arc component of $\ell_{0, n}^{j} \in \mathcal{C}_{0, n}$, for some $j \in \mathbb{Z}_{+}$, then $A \cap E_{0}$ contains at most one point with positive 0 -level. 
Proof Let $\ell_{0, n}^{j}$ be some link of $\mathcal{C}_{0, n}$ and let $A$ be an arc component of that link. Suppose that $A \cap E_{0}$ contains more than one point with positive 0 -level, and let $[\bar{x}],[\bar{y}] \in A \cap E_{0}$ be such that $l_{x}=L_{0}[\bar{x}]>0$ and $l_{y}=L_{0}[\bar{y}]>0$ and such that there are no 0 -points with positive 0 -level between $[\bar{x}]$ and $[\bar{y}]$. Without loss of generality assume that $l_{x}<l_{y}$. Consider $\pi_{l}(A)$, and let $B$ be the interval with endpoints $x^{\prime}=\vec{x}_{-l_{y}}$ and $y^{\prime}=\vec{y}_{-l_{y}}=\vec{c}_{0}$. Since there are no 0 -points between $[\bar{x}]$ and $[\bar{y}]$ with 0 -level greater than 0 , then clearly we have $\left.T_{s}^{m}\right|_{B}$ is monotone for all $m \leq l_{y}$. Since $T_{s}^{l_{y}-l_{x}}\left(x^{\prime}\right)=\vec{c}_{0}$ we have that $B$ is a maximal interval of monotonicity for $T_{s}^{l_{y}-l_{x}+j}$ for all $j \leq l_{x}$. Thus $d\left(T_{s}^{l_{y}}\left(y^{\prime}\right), T_{s}^{l_{y}}\left(x^{\prime}\right)\right) \geq \delta$. This implies that $\pi_{0}(A)$ has diameter greater than $\delta$. But $A$ is an arc component of a link of $\mathcal{C}_{0, n}$ which has mesh less than $\delta$, a contradiction.

Since $\mathcal{C}_{p, n} \preceq \mathcal{C}_{0, n}$ and $E_{p} \subseteq E_{0}$, the above lemma implies that if $A$ is an arc component of $\ell_{p, n}^{j}$ for some $j$, then $A \cap E_{p}$ can contain at most one $p$-point with nonzero $p$-level. We summarize this in the following remark.

Remark 3.2 Let $\ell_{p, n}^{j} \in \mathcal{C}_{p, n}$, and let $A$ be an arc component of $\ell_{p, n}^{j}$. Then $A$ contains at most one point from $E_{p}$ with nonzero $p$-level. Moreover, $\partial A \subset \ell_{p, n}^{i}$ where $i \in\{j-1, j+1\}$ if, and only if, $A$ contains a $p$-point with nonzero $p$-level.

Lemma 3.3 If $A$ is an arc component of $\ell_{p, n}^{j}$ such that $h^{-1}(A)$ contains at least one $q$-point with nonzero $q$-level, then $A$ contains a $p$-point with nonzero $p$-level. Moreover, $h^{-1}(A)$ contains precisely one $q$-point with nonzero $q$-level.

Proof Notice that we have

$$
\mathcal{C}_{q, m}^{\prime} \preceq h^{-1}\left(\mathcal{C}_{p, n}\right) \preceq \mathcal{C}_{g, e}^{\prime} .
$$

Let $A^{\prime}=h^{-1}(A)$, and suppose that $\left[\bar{x}^{\prime}\right] \in A^{\prime}$ is a $q$-point with nonzero $q$-level. Pick $r, u \in \mathbb{N}$ such that

$$
\left[\bar{x}^{\prime}\right] \in \ell_{q, m}^{\prime r} \subseteq h^{-1}\left(\ell_{p, n}^{j}\right) \subseteq \ell_{g, e}^{\prime u} .
$$

Since $E_{q}^{\prime} \subseteq E_{g}^{\prime},\left[\bar{x}^{\prime}\right]$ is also a $g$-point with nonzero $g$-level. Since each arc component of a link of $\mathcal{C}_{g, e}^{\prime}$ contains at most one $g$-point, we see that $\left[\bar{x}^{\prime}\right]$ is the unique $g$-point on its arc-component of $\ell_{g, e}^{\prime u}$, and therefore, the unique $q$-point on $h^{-1}(A)$ with nonzero $q$-level.

Let $B^{\prime}$ be the arc-component of $\ell_{g, e}^{\prime u}$ containing $A^{\prime}$, and let $k_{0}, k_{1} \in \mathbb{N}$ with $k_{0}<k_{1}$ minimal such that

$$
B^{\prime} \subset \bigcup_{y=k_{0}}^{k_{1}} \ell_{q, m}^{\prime y} .
$$


We see that $k_{0} \leq r \leq k_{1}$. Without loss of generality, assume that $B_{1}^{\prime}=A^{\prime} \cap \ell_{q, m}^{\prime r}=$ $B^{\prime} \cap \ell_{q, m}^{\prime r}$ has $\partial B_{1}^{\prime} \subset \ell_{q, m}^{\prime r-1}$. Then, if $r-1 \geq k_{0}$, since $B^{\prime}$ contains only one $g$-point, namely $\left[\bar{x}^{\prime}\right]$, we see that $B_{2}^{\prime}=A^{\prime} \cap\left(\ell_{q, m}^{\prime r} \cup \ell_{q, m}^{\prime r-1}\right)$ has $\partial B_{2}^{\prime} \subset \ell_{q, m}^{\prime r-2}$. Continuing in this manner we see that

$$
B_{j}^{\prime}=A^{\prime} \cap\left(\bigcup_{y=0}^{j-1} \ell_{q, m}^{\prime r-y}\right)
$$

has $\partial B_{j}^{\prime} \subset \ell_{q, m}^{r-j}$ for all $j$ with $r-j \geq k_{0}$. Since we have that

$$
\mathcal{C}_{q, m}^{\prime} \preceq h^{-1}\left(\mathcal{C}_{p, n}\right) \preceq \mathcal{C}_{g, e}^{\prime}
$$

there is some minimal $k$ such that $\partial B_{k}^{\prime} \cap h^{-1}\left(\ell_{p, n}^{j}\right)=\varnothing$. Since $\partial B_{k}^{\prime} \subset \ell_{q, m}^{\prime r-k}$, and since the chain $\mathcal{C}_{q, m}^{\prime}$ refines $h^{-1}\left(\mathcal{C}_{p, n}\right)$, there is a link, $\ell_{p, n}^{v}$ such that $\partial B_{k}^{\prime} \subset \ell_{q, m}^{\prime r-k} \subset$ $h^{-1}\left(\ell_{p, n}^{v}\right)$. Since $k$ was chosen minimal we see that $v \in\{j+1, j-1\}$. Without loss of generality assume that $v=j-1$. Thus there is an arc, $B_{k}=h\left(B_{k}^{\prime}\right)$, containing $A$ with $\partial B_{k} \subset \ell_{p, n}^{j-1}$. So $B_{k} \cap \ell_{p, n}^{j}=A$ has $\partial A \subset \ell_{p, n}^{j-1}$. This implies that $A$ has a $p$-point with nonzero $p$-level in $\ell_{p, n}^{j}$.

\subsection{Definition and basic properties of $\boldsymbol{h}_{q, p}$}

From the previous lemma we see that every $\left[\bar{x}^{\prime}\right] \in E_{q}^{\prime}$ with nonzero $q$-level is mapped to an arc component of $\ell_{p, n}^{x}$ containing a $p$-point with nonzero $p$-level.

We now define a map $h_{q, p}$ by first defining it on the $q$-points and then extending it to the rest of $C^{\prime}$. The map $h_{q, p}$ sends the $q$-point $\left[\bar{x}^{\prime}\right]$ with nonzero $q$-level to the unique $p$-point $[\bar{x}]$ that is on the same arc component of a link of $\mathcal{C}_{p, n}$ as $h\left(\left[\bar{x}^{\prime}\right]\right)$. In other words:

Definition 3.4 Let $\left[\bar{x}^{\prime}\right] \in E_{q}^{\prime}$ be such that $L_{q}\left(\left[\bar{x}^{\prime}\right]\right) \neq 0$, and let $h\left(\left[\bar{x}^{\prime}\right]\right)=[\bar{u}]$, for some $[\bar{u}] \in C$. Let $\ell_{q, m}^{x^{\prime}} \in \mathcal{C}_{q, m}^{\prime}$ be a link which contains $\left[\bar{x}^{\prime}\right]$, and let $\ell_{p, n}^{x} \in \mathcal{C}_{p, n}$ be a link such that $h\left(\ell_{q, m}^{x^{\prime}}\right) \subset \ell_{p, n}^{x}$. Then define $h_{q, p}\left(\left[\bar{x}^{\prime}\right]\right)=[\bar{x}] \in E_{p} \cap \ell_{p, n}^{x}$, where $\overleftarrow{x}_{-p-1}=\overleftarrow{u}_{-p-1}$, for some $\bar{x} \in[\bar{x}]$ and $\bar{u} \in[\bar{u}]$

Next, let $A^{\prime} \subset C^{\prime}$ be an arc with $\partial A^{\prime}=\left\{\left[\bar{x}^{\prime}\right],\left[\bar{y}^{\prime}\right]\right\}$ such that $\left[\bar{x}^{\prime}\right]$ and $\left[\bar{y}^{\prime}\right]$ are adjacent $q$-points with nonzero $q$-levels. Define $h_{q, p}\left(A^{\prime}\right)=A$ to be the arc with endpoints $h_{q, p}\left(\left[\bar{x}^{\prime}\right]\right)$ and $h_{q, p}\left(\left[\bar{y}^{\prime}\right]\right)$ such that $\left.h_{q, p}\right|_{A^{\prime}}$ is monotone.

By the previous lemma, $h_{q, p}: C^{\prime} \rightarrow C$ is a one-to-one function. The mapping $h_{q, p}: C^{\prime} \rightarrow C$ has been defined using the homeomorphism $h: K_{t} \rightarrow K_{s}$. Since $h^{-1}: K_{s} \rightarrow K_{t}$ is also a homeomorphism, we can define a mapping $h_{p, g}^{\prime}: C \rightarrow C^{\prime}$ in the same manner, using the homeomorphism $h^{-1}$ instead of $h$. Clearly $h_{p, g}^{\prime}$ has all the properties of $h_{q, p}$. Also, it is easy to see that $h_{p, g}^{\prime}\left(h_{q, p}\left(\left[\bar{x}^{\prime}\right]\right)\right)=\left[\bar{x}^{\prime}\right]$, for every $q$-point $\left[\bar{x}^{\prime}\right]$ with nonzero $q$-level. 
Next we show that if we have arcs $A^{\prime}$ and $D^{\prime}$ in $C^{\prime}$ with the same projection under $\pi_{q}$ and no $q$-points with nonzero $q$-level in the interior of $A^{\prime}$ and $D^{\prime}$, then the $h_{q, p}$-image of these arcs follows the same path through the links of $\mathcal{C}_{p, n}$ in the sense that if there is a link that $A=h_{q, p}\left(A^{\prime}\right)$ "turns" in, then $D=h_{q, p}\left(D^{\prime}\right)$ will also "turn" in that link. Notice that by assuming that $A^{\prime}$ and $D^{\prime}$ have no $q$-points with nonzero $q$-level, we are assuming that $A^{\prime}$ and $D^{\prime}$ "run straight" through the links of $\mathcal{C}_{q, m}^{\prime}$ that they "visit". Hence their images will follow the same path through the links of $\mathcal{C}_{p, n}$.

Lemma 3.5 Let $A^{\prime}$ and $D^{\prime}$ be arcs of $C^{\prime}$ with

(1) $\partial A^{\prime}, \partial D^{\prime} \subset E_{q}^{\prime}$ with nonzero $q$-level;

(2) $\pi_{q}\left(A^{\prime}\right)=\pi_{q}\left(D^{\prime}\right)$;

(3) if $\left[\bar{x}^{\prime}\right] \in E_{q}^{\prime} \cap\left(\operatorname{Int} A^{\prime} \cup \operatorname{Int} D^{\prime}\right)$ then $L_{q}\left[\bar{x}^{\prime}\right]=0$.

Let $h_{q, p}\left(A^{\prime}\right)=A$ and $h_{q, p}\left(D^{\prime}\right)=D$. Let $j$ be such that $(\partial A \cup \partial D) \cap \ell_{p, n}^{j}=\varnothing$ and $(A \cup D) \cap \ell_{p, n}^{j} \neq \varnothing$. Let $A_{j}=\ell_{p, n}^{j} \cap A$ and let $D_{j}=\ell_{p, n}^{j} \cap D$. Then $\partial A_{j} \subset \ell_{p, n}^{j-1}$ if, and only if, $\partial D_{j} \subset \ell_{p, n}^{j-1}$. Similarly, $\partial A_{j} \subset \ell_{p, n}^{j+1}$ if, and only if, $\partial D_{j} \subset \ell_{p, n}^{j+1}$.

Proof Let $a$ and $b$ be such that $h\left(\bigcup_{i=a}^{b} \ell_{q, m}^{i}\right) \subset \ell_{p, n}^{j}$ and $h\left(\ell_{q, m}^{\prime a-1}\right) \nsubseteq \ell_{p, n}^{j}$ and $h\left(\ell_{q, m}^{\prime b+1}\right) \nsubseteq \ell_{p, n}^{j}$. Let

$$
A_{j}^{\prime}=\left(\bigcup_{i=a}^{b} \ell_{q, m}^{i}\right) \cap A^{\prime} \quad \text { and } \quad D_{j}^{\prime}=\left(\bigcup_{i=a}^{b} \ell_{q, m}^{\prime i}\right) \cap D^{\prime} .
$$

Since $A_{j}^{\prime}$ and $D_{j}^{\prime}$ do not contain any $q$-points with nonzero $q$-level in their interiors, there are points $\left[\bar{u}^{\prime A}\right] \in \mathrm{Cl} A_{j}^{\prime} \cap \ell_{q, m}^{\prime a-1}$ and $\left[\bar{u}^{\prime D}\right] \in \mathrm{Cl} D_{j}^{\prime} \cap \ell_{q, m}^{\prime a-1}$, and there are also points $\left[\bar{v}^{\prime A}\right] \in \mathrm{Cl} A_{j}^{\prime} \cap \ell_{q, m}^{\prime b+1}$ and $\left[\bar{v}^{\prime D}\right] \in \mathrm{Cl} D_{j}^{\prime} \cap \ell_{q, m}^{\prime b+1}$. Since $h\left(\ell_{q, m}^{\prime a-1}\right) \nsubseteq \ell_{p, n}^{j}$ and $h\left(\ell_{q, m}^{\prime b+1}\right) \nsubseteq \ell_{p, n}^{j}$, then either $h\left(\ell_{q, m}^{\prime a-1}\right) \subset \ell_{p, n}^{j-i}$ and $h\left(\ell_{q, m}^{\prime b+1}\right) \subset \ell_{p, n}^{j+i}$, with $i \in\{-1,1\}$, or $h\left(\ell_{q, m}^{\prime a-1}\right) \subset \ell_{p, n}^{j-i}$ and $h\left(\ell_{q, m}^{\prime b+1}\right) \subset \ell_{p, n}^{j-i}$, with $i \in\{-1,1\}$. The result follows.

Next we show that if $A^{\prime}$ and $D^{\prime}$ are arcs with the same $q$-projection and again no $q$-points in their interiors with nonzero $q$-level, then their $h_{q, p}$-images will have the same number of $p$-points and the arrangement of these $p$-points will be similar.

Lemma 3.6 Let $A^{\prime}$ and $D^{\prime}$ be arcs of $C^{\prime}$ with

(1) $\partial A^{\prime}, \partial D^{\prime} \subset E_{q}^{\prime}$ with nonzero $q$-level;

(2) $\pi_{q}\left(A^{\prime}\right)=\pi_{q}\left(D^{\prime}\right)$;

(3) if $\left[\bar{x}^{\prime}\right] \in E_{q}^{\prime} \cap\left(\operatorname{Int} A^{\prime} \cup \operatorname{Int} D^{\prime}\right)$ then $L_{q}\left[\bar{x}^{\prime}\right]=0$.

If $h_{q, p}\left(A^{\prime}\right) \cap E_{p}=\left\{\left[\bar{x}^{0}\right],\left[\bar{x}^{1}\right], \ldots,\left[\bar{x}^{k}\right]\right\}$ and $h_{q, p}\left(D^{\prime}\right) \cap E_{p}=\left\{\left[\bar{y}^{0}\right],\left[\bar{y}^{1}\right], \ldots,\left[\bar{y}^{l}\right]\right\}$ then $k=l$ and $\left[\bar{x}^{i}\right] \in \ell_{p, n}^{y^{i}}$ or $\left[\bar{x}^{i}\right] \in \ell_{p, n}^{y^{k-i}}$, for $i=0, \ldots, k$. 
Proof Let $A^{\prime}$ and $D^{\prime}$ be such arcs. Let $A=h_{q, p}\left(A^{\prime}\right)$ and $D=h_{q, p}\left(D^{\prime}\right)$. Suppose, on the contrary, that $k>l$. Then there exists a link $\ell_{p, n}^{j}$ of $\mathcal{C}_{p, n}$ which contains a $p$-point $[\bar{u}] \in A$ and which does not contain a $p$-point in $D$. By the previous lemma, each arc $A_{j}$ enters and exits the link $\ell_{p, n}^{j}$ from the same side as the arc $D_{j}$. This contradicts the assumption that one arc contains a $p$-point and one does not. Thus $k=l$. The fact that either $\left[\bar{x}^{i}\right] \in \ell_{p, n}^{y^{i}}$ or $\left[\bar{x}^{i}\right] \in \ell_{p, n}^{y^{k-i}}$, for $i=0, \ldots, k$, follows immediately.

Definition 3.7 Let $\ell_{p, n}^{j_{0}}, \ell_{p, n}^{j_{1}}, \ldots, \ell_{p, n}^{j_{k}}$ be the links in $\mathcal{C}_{p, n}$ that are successively visited by an $\operatorname{arc} A$. (Hence $\ell_{p, n}^{j_{i}} \neq \ell_{p, n}^{j_{i+1}}, \ell_{p, n}^{j_{i}} \cap \ell_{p, n}^{j_{i}+1} \neq \varnothing$ and $\ell_{p, n}^{j_{i}}=\ell_{p, n}^{j_{i}+2}$ is possible if $A$ turns in $\ell_{p, n}^{j_{i}+1}$.) We call $A$ p-link-symmetric if for $i=0, \ldots, k, \ell_{p, n}^{j_{i}}=\ell_{p, n}^{j_{k-i}}$.

Note that every $p$-symmetric arc is $p$-link-symmetric by definition, but there are $p$-link-symmetric arcs which are not $p$-symmetric. This occurs if $A$ turns both at $\ell_{p, n}^{j_{i}}$ and $\ell_{p, n}^{j_{k-i}}$, but the $p$-point of $A$ in $\ell_{p, n}^{j_{i}}$ has different $p$-level than the $p$-point of $A$ in $\ell_{p, n}^{j_{k-i}}$. Note also that if $A^{\prime}$ and $D^{\prime}$ from the previous lemma (Lemma 3.6) are such that additionally $A^{\prime} \cap D^{\prime}=\left[\bar{x}^{\prime}\right] \in E_{q}^{\prime}$, then lemma states that $h_{q, p}\left(A^{\prime}\right)$ is $p$-link-symmetric.

\subsection{Image of $q$-levels}

Next we show that all $q$-points in $C^{\prime}$ of some fixed level, say $a$, map to $p$-points of some other fixed level, say $b$, in $C$. This fact will imply Ingram's Conjecture.

Let $A^{\prime}$ be a $q$-symmetric arc with $\partial A \in E_{q}^{\prime}$ and let $A^{\prime} \cap E_{q}^{\prime}=\left\{\left[\bar{x}^{\prime 0}\right], \ldots,\left[\bar{x}^{\prime l}\right]\right\}$. Then $L_{q}\left[\bar{x}^{\prime i}\right]=L_{q}\left[\bar{x}^{\prime l-i}\right]$, for every $i \in\{0, \ldots, l\}$. Let $A=h_{q, p}\left(A^{\prime}\right)$ and let $A \cap E_{p}=$ $\left\{\left[\bar{x}^{0}\right], \ldots,\left[\bar{x}^{k}\right]\right\}$. Then, by Lemma $3.6, A$ is $p$-link-symmetric, ie, $\left[\bar{x}^{i}\right] \in \ell_{p, n}^{x^{k-i}}$, for $i=0, \ldots, k$. A natural question is if such an $A$ is $p$-symmetric, ie, if the following, much stronger property, holds:

$$
L_{p}\left[\bar{x}^{i}\right]=L_{p}\left[\bar{x}^{k-i}\right]
$$

for $i=0, \ldots, k$. The answer is yes, but the proof is not straightforward. Let us explain the major problem. Let $U$ be an arc such that for $U \cap E_{p}=\left\{\left[\bar{y}^{0}\right], \ldots,\left[\bar{y}^{j}\right]\right\}$ we have $L_{p}\left[\bar{y}^{i}\right]=L_{p}\left[\bar{y}^{j-i}\right]$, for $i=1, \ldots, j-1$. It is clear that if $\left[\bar{y}^{0}\right] \in \ell_{p, n}^{y^{j}}$ implied $L_{p}\left[\bar{y}^{0}\right]=L_{p}\left[\bar{y}^{j}\right]$, then a proof of (1) would be simple. Unfortunately, there are tent map inverse limits for which the above implication does not hold, ie, there are spaces which contain arcs $U$ such that $U \cap E_{p}=\left\{\left[\bar{y}^{0}\right], \ldots,\left[\bar{y}^{j}\right]\right\}$ with $L_{p}\left[\bar{y}^{i}\right]=L_{p}\left[\bar{y}^{j-i}\right]$, for $i=1, \ldots, j-1,\left[\bar{y}^{0}\right] \in \ell_{p, n}^{y^{j}}$ and $L_{p}\left[\bar{y}^{0}\right] \neq L_{p}\left[\bar{y}^{j}\right]$. Such arcs we call quasi-psymmetric with respect to $\mathcal{C}_{p, n}$ (see Definition 4.1). Since we can choose $p$ and $n$ to generate chains with arbitrarily small mesh, we see that quasi- $p$-symmetric arcs will not be an issue unless we are in a situation that allows $p$-points with different 
$p$-levels to be arbitrarily close. This can only happen when $1 / 2$ is prerecurrent. The subfamily of nonrecurrent tent maps which have prerecurrent critical points is large, and among these there are many nonrecurrent tent maps which have quasi-symmetric arcs in their inverse limit.

To get a proof of (1) in this case we give a much more detailed description of the structure of the composant $C$. This part is technical and would break the flow of the main argument. Therefore, we put all of the properties related to the structure of the composant $C$ which contains quasi- $p$-symmetric arcs for every $p$ in the Appendix. It suffices to say that nonrecurrent tent map inverse limits either contain quasi- $p$-symmetric arcs with respect to $\mathcal{C}_{p, n}$ for finitely many $p \in \mathbb{Z}_{+}$, or they contain quasi- $p$-symmetric arcs with respect to $\mathcal{C}_{p, n}$ for infinitely many $p \in \mathbb{Z}_{+}$. In the first case there exists an integer $S$ (as defined in Remark 4.7), such that for all $n \geq S$ and $p \in \mathbb{Z}_{+}$there are no quasi- $p$-symmetric arcs with respect to $\mathcal{C}_{p, n}$.

It is easy to see that in the second case, there exist quasi- $p$-symmetric arcs with respect to $\mathcal{C}_{p, n}$ for every $p \in \mathbb{Z}_{+}$and $n \in \mathbb{N}$ (Lemma 4.2). In this case we prove the following proposition in the Appendix:

Proposition 3.8 For every quasi-q-symmetric $\operatorname{arc} A^{\prime}$ of $C^{\prime}$, the $\operatorname{arc} h_{q, p}\left(A^{\prime}\right)$ is not p-symmetric.

Armed with Proposition 3.8 we can prove the main results of this section. Specifically we show that $q$-levels map to $p$-levels.

We prove the main result of this section in two steps. First in the absence of quasisymmetric arcs, and then in the general case. Recall that for this section we assume that $q, p, g \in \mathbb{Z}_{+}, m, e \geq S^{\prime}$ and $n \geq S$ are such that

$$
h\left(\mathcal{C}_{q, m}^{\prime}\right) \preceq \mathcal{C}_{p, n} \preceq h\left(\mathcal{C}_{g, e}^{\prime}\right),
$$

where $S, S^{\prime} \in \mathbb{N}$ are large enough so that all technical requirements of Remark 4.7 from the Appendix are satisfied.

Theorem 3.9 Let $K_{t}$ and $K_{s}$ be such that there are no quasi-q-symmetric arcs with respect to $\mathcal{C}_{q, m}^{\prime}$ and no quasi-p-symmetric arcs with respect to $\mathcal{C}_{p, n}$. Let $\left[\bar{x}^{\prime}\right],\left[\bar{y}^{\prime}\right] \in E_{q}^{\prime}$ be such that $L_{q}\left[\bar{x}^{\prime}\right]=L_{q}\left[\bar{y}^{\prime}\right]$. Then $L_{p}[\bar{x}]=L_{p}[\bar{y}]$, where $[\bar{x}]=h_{q, p}\left(\left[\bar{x}^{\prime}\right]\right)$ and $[\bar{y}]=h_{q, p}\left(\left[\bar{y}^{\prime}\right]\right)$.

Proof Suppose, on the contrary, that $\left[\bar{x}^{\prime}\right],\left[\bar{y}^{\prime}\right] \in E_{q}^{\prime}$ are the closest $q$-points such that $L_{q}\left[\bar{x}^{\prime}\right]=L_{q}\left[\bar{y}^{\prime}\right]$ and $L_{p}[\bar{x}] \neq L_{p}[\bar{y}]$. Then there is no $q$-point between $\left[\bar{x}^{\prime}\right]$ and $\left[\bar{y}^{\prime}\right]$ which has the same $q$-level as the point $\left[\bar{x}^{\prime}\right]$. Denote by $A^{\prime}$ the arc between 
$\left[\bar{x}^{\prime}\right]$ and $\left[\bar{y}^{\prime}\right], A^{\prime} \cap E_{q}^{\prime}=\left\{\left[\bar{x}^{\prime}\right]=\left[\bar{x}^{\prime 0}\right], \ldots,\left[\bar{x}^{\prime l}\right]=\left[\bar{y}^{\prime}\right]\right\}$. Since between $\left[\bar{x}^{\prime}\right]$ and $\left[\bar{y}^{\prime}\right]$ there are no $q$-points with $q$-levels equal to $L_{q}\left[\bar{x}^{\prime}\right]$, there is a $q$-point $\left[\bar{z}^{\prime}\right]$ between them such that $L_{q}\left[\bar{z}^{\prime}\right]=\max \left\{L_{q}\left[\bar{x}^{\prime i}\right]: i=0, \ldots, l\right\},\left[\bar{z}^{\prime}\right]=\left[\bar{x}^{\prime l / 2}\right]$, and $L_{q}\left[\bar{x}^{\prime i}\right]=L_{q}\left[\bar{x}^{\prime l-i}\right]$, for every $i \in\{0, \ldots, l\}$. Therefore, $L_{p}\left[\bar{x}^{i}\right]=L_{p}\left[\bar{x}^{l-i}\right]$, for every

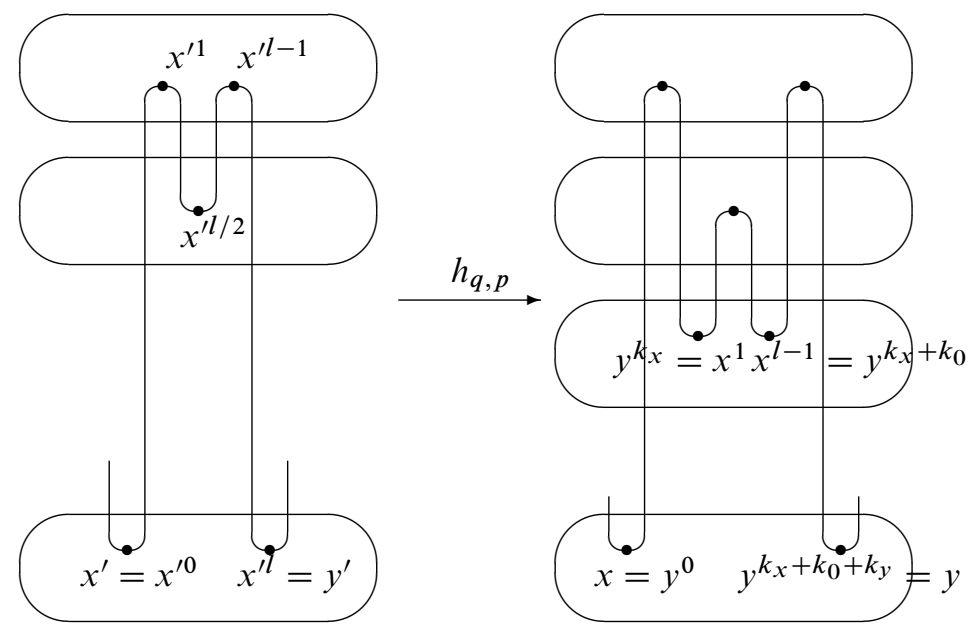

Figure 3: The configuration in the proof of Theorem 3.9

$i \in\{1, \ldots, l-1\}$, where $\left[\bar{x}^{i}\right]=h_{q, p}\left(\left[\bar{x}^{\prime i}\right]\right)$, because we chose $\left[\bar{x}^{\prime}\right]$ and $\left[\bar{y}^{\prime}\right]$ to be the closest two points where this fails. Denote by $A$ the arc between $[\bar{x}]$ and $[\bar{y}]$, by $A_{0}$ the arc between $\left[\bar{x}^{1}\right]$ and $\left[\bar{x}^{l-1}\right]$, by $A_{x}$ the arc between $[\bar{x}]=\left[\bar{x}^{0}\right]$ and $\left[\bar{x}^{1}\right]$ and by $A_{y}$ the arc between $\left[\bar{x}^{l-1}\right]$ and $[\bar{y}]=\left[\bar{x}^{l}\right]$. The $\operatorname{arc} A_{0}$ is $p$-symmetric. Let $A \cap E_{p}=$ $\left\{[\bar{x}]=\left[\bar{y}^{0}\right], \ldots,\left[\bar{y}^{k_{x}}\right]=\left[\bar{x}^{1}\right], \ldots,\left[\bar{x}^{l-1}\right]=\left[\bar{y}^{k_{x}+k_{0}}\right], \ldots,\left[\bar{y}^{k_{x}+k_{0}+k_{y}}\right]=[\bar{y}]\right\}$. Note

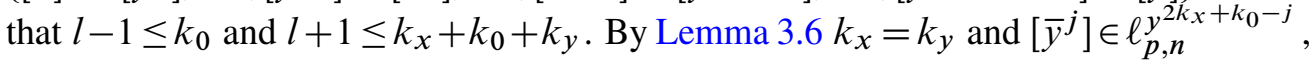
for $j=1, \ldots, k_{x}$. Since $K_{s}$ does not contain any quasi-p-symmetric arcs, then $L_{p}\left[\bar{y}^{j}\right]=L_{p}\left[\bar{y}^{2 k_{x}+k_{0}-j}\right]$, for every $j \in\left\{0, \ldots, k_{x}\right\}$, which contradicts the assumption that $L_{p}[\bar{x}] \neq L_{p}[\bar{y}]$.

We next prove the previous theorem without the assumption that there are no quasisymmetric arcs in the inverse limit. We use Proposition 3.8 (proved in the Appendix).

Theorem 3.10 Let $\left[\bar{x}^{\prime}\right],\left[\bar{y}^{\prime}\right] \in E_{q}^{\prime}$ be such that $L_{q}\left[\bar{x}^{\prime}\right]=L_{q}\left[\bar{y}^{\prime}\right]$. Then $L_{p}[\bar{x}]=L_{p}[\bar{y}]$, where $[\bar{x}]=h_{q, p}\left(\left[\bar{x}^{\prime}\right]\right)$ and $[\bar{y}]=h_{q, p}\left(\left[\bar{y}^{\prime}\right]\right)$. 
Proof Suppose the theorem is not true, and let $\left[\bar{x}^{\prime}\right]$ and $\left[\bar{y}^{\prime}\right]$ be the closest $q$-points for which the theorem is false. Then the arc between these points, call it $A^{\prime}$, is $q$-symmetric.

Suppose that $h_{q, p}\left(A^{\prime}\right)$ is not $p$-symmetric. Let $A^{\prime} \cap E_{q}^{\prime}=\left\{\left[\bar{x}^{\prime}\right]=\left[\bar{x}^{\prime 0}\right], \ldots,\left[\bar{x}^{\prime k}\right]=\right.$ $\left.\left[\bar{y}^{\prime}\right]\right\}$, and let $A=h_{q, p}\left(A^{\prime}\right)$. Let $A \cap E_{p}=\left\{\left[\bar{y}^{0}\right], \ldots,\left[\bar{y}^{l}\right]\right\}$, and suppose there is some $j \in\left\{0,1, \ldots, \frac{l}{2}-1\right\}$ such that $L_{p}\left[\bar{y}^{j}\right] \neq L_{p}\left[\bar{y}^{l-j}\right]$. Without loss of generality, assume $j$ is the largest with this property. Let $D$ be the arc from $\left[\bar{y}^{j}\right]$ to $\left[\bar{y}^{l-j}\right]$. Then $D$ is a quasi- $p$-symmetric arc, and let $D^{\prime}=h_{p, g}^{\prime}(D)$. Let $A^{\prime} \cap E_{g}^{\prime}=\left\{\left[\bar{z}^{\prime 0}\right], \ldots,\left[\bar{z}^{\prime d}\right]\right\}$. Since $E_{q}^{\prime} \subseteq E_{g}^{\prime}$ we see that $L_{g}\left[\bar{z}^{\prime i}\right]=L_{g}\left[\bar{z}^{\prime d-i}\right]$ for all $i \in\{0, \ldots, d\}$, but since $D$ is quasi-p-symmetric, by Proposition $3.8, D^{\prime}$ cannot be $q$-symmetric. Since $D^{\prime}$ is an arc centered at $\left[\bar{z}^{\prime d / 2}\right]$ and contained in $A^{\prime}$, it is a $q$-symmetric arc, a contradiction.

Therefore, $A=h_{q, p}\left(A^{\prime}\right)$ is $p$-symmetric, but by our assumption the boundary points of $A,[\bar{x}]=h_{q, p}\left[\bar{x}^{\prime}\right]$ and $[\bar{y}]=h_{q, p}\left[\bar{y}^{\prime}\right]$, have different $p$-levels, a contradiction. $\square$

Combining the previous two theorems we have:

Corollary 3.11 If $l$ and $k$ are such that $h_{q, p}\left(\left[\bar{x}^{\prime}\right]\right) \in E_{p, k}$ for $\left[\bar{x}^{\prime}\right] \in E_{q, l}^{\prime}$ then $h_{q, p}\left(E_{q, l}^{\prime}\right)=E_{p, k}$.

Proof By our assumptions on $k$ and $l$ and by Theorem 3.10, we see immediately that $h_{q, p}\left(E_{q, l}^{\prime}\right) \subseteq E_{p, k}$.

Let $[\bar{y}] \in E_{p, k}$. Since $h_{p, g}^{\prime}\left(h_{q, p}\left(\left[\bar{x}^{\prime}\right]\right)\right)=\left[\bar{x}^{\prime}\right] \in E_{q, l}^{\prime}=E_{g, q-g+l}^{\prime}$, then $h_{p, g}^{\prime}([\bar{y}])=$ $\left[\bar{z}^{\prime}\right] \in E_{g, q-g+l}^{\prime}$. Since $h_{p, g}^{\prime}\left(h_{q, p}\left(\left[\bar{z}^{\prime}\right]\right)\right)=\left[\bar{z}^{\prime}\right]$ and $h_{p, g}^{\prime}$ is an injection, we also have $[\bar{y}]=h_{q, p}\left(\left[\bar{z}^{\prime}\right]\right)$. Thus $h_{q, p}\left(E_{q, l}\right)=E_{p, k}$.

\section{3 $h_{q, p}$ preserves the order of the levels}

In this subsection, we consider how $h_{q, p}$ maps the various $q$-levels of $E_{q}^{\prime}$. We show that there is some $a \in \mathbb{N}$ such that

$$
h_{q, p}\left(E_{q, j}^{\prime}\right)=E_{p, a+j}
$$

for all $j \in \mathbb{N}$.

From now on, let $a \in \mathbb{N}$ be such that $h_{q, p}\left(E_{q, 1}^{\prime}\right)=E_{p, a+1}$.

Remark 3.12 Recall, for this section we have assumed that $q, p, g \in \mathbb{Z}_{+}, m, e \geq$ $S^{\prime}$ and $n \geq S$ are such that $h\left(\mathcal{C}_{q, m}^{\prime}\right) \preceq \mathcal{C}_{p, n} \preceq h\left(\mathcal{C}_{g, e}^{\prime}\right)$. Let $b=q-g$. Since $h_{p, g}^{\prime}\left(E_{p, a+1}\right) \subseteq E_{q, 1}^{\prime}$, then, by Corollary 3.11, $h_{p, g}^{\prime}\left(E_{p, a+1}\right)=E_{q, 1}^{\prime}$. Since by definition $E_{q, 1}^{\prime}=E_{g, q-g+1}^{\prime}=E_{g, b+1}^{\prime}$, we have $h_{p, g}^{\prime}\left(E_{p, a+1}\right)=E_{g, b+1}^{\prime}$. 
Lemma 3.13 For each $u \in \mathbb{N}$ let $i_{u} \in \mathbb{Z}_{+}$be defined such that $h_{p, g}^{\prime}\left(E_{p, a+u}\right)=$ $E_{g, b+i_{u}}^{\prime}$. Then the subsequences $\left(i_{2 u}\right)_{u \in \mathbb{N}}$ and $\left(i_{2 u+1}\right)_{u \in \mathbb{N}}$ are increasing.

Proof We prove the lemma for the case of $2 u$. Suppose by way of contradiction that $u$ is minimal with $i_{2 u}>i_{2 u+2}$. Lemma 2.16 implies that there is a $p$-symmetric arc $B \subset C$ with $\partial B=\{[\bar{x}],[\bar{y}]\}$ and $L_{p}[\bar{x}]=2 u=L_{p}[\bar{y}]$ and $L_{p}\left[\bar{\chi}^{B}\right]=2 u+2$. Then $h_{p, g}^{\prime}(B)=B^{\prime}$ is a $g$-symmetric arc, by Proposition 3.8, but $L_{g}\left(h_{p, g}^{\prime}[\bar{x}]\right)=i_{2 u}=$ $L_{g}\left(h_{p, g}^{\prime}[\bar{y}]\right)$ while $L_{g}\left[\bar{\chi}^{B^{\prime}}\right]=L_{g}\left(h_{p, g}^{\prime}\left[\bar{\chi}^{B}\right]\right)=i_{2 u+2}<i_{2 u}$, a contradiction. The case that $i_{2 u+1}>i_{2 u+3}$ for some $u$ leads to a contradiction in a similar manner.

We divide the main result of this section into two cases:

(1) $c_{3}=0$ or $c_{3}^{\prime}=0$.

(2) Both $c_{3}=1$ and $c_{3}^{\prime}=1$.

Theorem 3.14 If $c_{3}=0$, then for each $j \in \mathbb{N}, E_{q, j}^{\prime}$ is mapped by $h_{q, p}$ to $E_{p, a+j}$.

Proof Suppose the theorem is false. Choose the least $j>1$ such that $h_{q, p}\left(E_{q, j}^{\prime}\right)=$ $E_{p, a+l}$ with $l \neq j$.

First suppose that $l<j$. Since $h_{q, p}\left(E_{q, i}^{\prime}\right)=E_{p, a+i}$ for $i=1, \ldots, j-1$ and $h_{q, p}$ is an injection, then $a+l \leq a$. To see this, suppose instead that $a<a+l<a+j$. Then by our assumption on $j$ we would have $h_{q, p}\left(E_{q, j}^{\prime}\right)=E_{p, a+l}=h_{q, p}\left(E_{q, l}^{\prime}\right)$. Thus $h_{q, p}$ is not injective, a contradiction.

By Lemma 2.15 there is a $p$-bridge which will shift under the $a+l$-power of the shift map to a $p$-symmetric arc $B \subset C$ with $\partial B=\{[\bar{x}],[\bar{y}]\}, L_{p}[\bar{x}]=a+l=L_{p}[\bar{y}]$ and $L_{p}\left[\bar{\chi}^{B}\right]=a+j-1$ (recall that $l \leq 0$ and $j-1 \geq 1$ ). Let $B^{\prime}=h_{p, g}^{\prime}(B)$. Then, by Theorem 3.10, $B^{\prime}$ is a $g$-symmetric arc whose boundary points have level $b+j$ while its center has level $b+j-1$, where $b=q-g$ as defined in Remark 3.12, a contradiction. So we have instead that $l>j$.

Let $m_{k} \in \mathbb{Z}_{+}$be such that $h_{p, g}^{\prime}\left(E_{p, a+j+k}\right)=E_{g, m_{k}}^{\prime}$ for $0 \leq k \leq l-j-1$. Since $h_{p, g}^{\prime}\left(h_{q, p}\left(E_{q, i}^{\prime}\right)\right)=E_{g, b+i}^{\prime}$, for all $i \in \mathbb{N}$, we have that

$$
h_{p, g}^{\prime}\left(h_{q, p}\left(E_{q, j}^{\prime}\right)\right)=h_{p, g}^{\prime}\left(E_{p, a+l}\right)=E_{g, b+j}^{\prime} .
$$

Also, since $j$ was chosen to be minimal,

$$
h_{p, g}^{\prime}\left(E_{p, a+i}\right)=E_{g, b+i}^{\prime}
$$

for all $i, 1 \leq i \leq j-1$. Thus, for all $k, 0 \leq k \leq l-j-1$, either $m_{k} \leq b$ or $m_{k}>b+j$. 
Suppose first that there is some $k, 0 \leq k \leq l-j-1$ with $m_{k}>b+j$. Then by Lemma 2.15, there is a $p$-symmetric arc $B \subset C$ with $\partial B=\{[\bar{x}],[\bar{y}]\}$, such that $L_{p}[\bar{x}]=a+j+k=L_{p}[\bar{y}]$ and $L_{p}\left[\bar{\chi}^{B}\right]=a+l$, where obviously $l>j+k$. Since $h_{p, g}^{\prime}\left(E_{p, a+j+k}\right)=E_{g, m_{k}}^{\prime}$ we see that, by Theorem $3.10, B^{\prime}=h_{p, g}^{\prime}(B)$ is a $g$-symmetric arc with boundary points in $E_{g, m_{k}}^{\prime}$ but center in $E_{g, b+j}^{\prime}$. This is a contradiction because we assumed that $m_{k}>b+j$. Thus $m_{k} \leq b$ for all $0 \leq k \leq$ $l-j-1$.

By Lemma 2.15 there is a $p$-symmetric arc $B \subset C$ such that the boundary points of $B$ have $p$-level $a+j-1$ and the center of $B$ has $p$-level $a+j$. Let $B^{\prime}=h_{p, g}^{\prime}(B)$. Then $B^{\prime}$ is a $g$-symmetric arc with boundary points in $E_{g, b+j-1}^{\prime}$ and center in $E_{g, m_{0}}^{\prime}$. Since $j \in \mathbb{N}$, we see that $b+j-1 \geq b \geq m_{0}$, a contradiction. Thus in either case we see that $h_{q, p}\left(E_{q, j}^{\prime}\right)=E_{p, a+j}$.

If instead $c_{3}^{\prime}=0$ we can use $h^{-1}$ and get the same result, so we now turn to the case that both $c_{3}=1$ and $c_{3}^{\prime}=1$.

For the remainder of this section we suppose $c_{3}=1=c_{3}^{\prime}$. Let $n^{\prime}=\min \{i \in \mathbb{N}$ : $\left.c_{2 i+1}^{\prime}=0\right\}$, and let $n=\min \left\{i \in \mathbb{N}: c_{2 i+1}=0\right\}$. Without loss of generality assume that $n^{\prime} \geq n$. Our goal now is to prove that $h_{q, p}$ maps the level $E_{q, j}^{\prime}$ to $E_{p, a+j}$ for all $j \in \mathbb{N}$. This is accomplished in several small steps.

Lemma 3.15 Let $u \in \mathbb{Z}_{+}$. Then there is some $l \geq 0$ such that $h_{q, p}\left(E_{q, 2 u+1}^{\prime}\right)=$ $E_{p, a+2 u+1+l}$.

Proof Let $B^{\prime} \subset C^{\prime}$ be a $q$-symmetric arc with $L_{q}\left[\bar{\chi}^{B^{\prime}}\right]=3$ and $L_{q}\left[\bar{x}^{\prime}\right]=1$ for $\left[\bar{x}^{\prime}\right] \in \partial B^{\prime}$. Then $B=h_{q, p}\left(B^{\prime}\right)$ is a $p$-symmetric arc with $L_{p}[\bar{x}]=a+1$ for all $[\bar{x}] \in \partial B$, and so $L_{p}\left[\bar{\chi}^{B}\right]>a+1$. If $L_{p}\left[\bar{\chi}^{B}\right]=a+2$ then we have a contradiction because $c_{3} \neq 0$ and by Remark 2.18 there exists only one $p$-symmetric arc with this property. So we see that $L_{p}\left[\bar{\chi}^{B}\right] \geq a+3$, and hence $h_{q, p}\left(E_{q, 3}^{\prime}\right)=E_{p, a+3+l}$ with $l \geq 0$. By induction the lemma follows.

This lemma combined with Lemma 3.13 gives that, in the case of $c_{3}=1=c_{3}^{\prime}$, each of the odd levels, $E_{q, 2 u+1}^{\prime}$, map to a level no lower than our goal of $E_{p, a+2 u+1}$. Similarly we have the following:

Lemma 3.16 Let $u \in \mathbb{Z}_{+}$. Then there is an $l \geq 0$ such that $h_{q, p}\left(E_{q, 2 u}^{\prime}\right)=E_{p, a+2 u+l}$.

Proof We show that $h_{q, p}\left(E_{q, 2}^{\prime}\right)$ is sent to $E_{p, a+2+l}$ with $l \geq 0$. The result will follow by induction and the fact that $c_{3}^{\prime}=1$. 
Suppose that $h_{q, p}\left(E_{q, 2}^{\prime}\right)=E_{p, a-u}$ for some $u \in \mathbb{Z}_{+}$. By the previous lemma we have for all $v \in \mathbb{Z}_{+}$there is some $k_{v} \in \mathbb{Z}_{+}$such that

$$
h_{q, p}\left(E_{q, 2 v+1}^{\prime}\right)=E_{p, a+2 v+1+k_{v}} .
$$

Let $v$ be chosen such that $2 v+1=2 n^{\prime}-1$, where $n^{\prime}$ is as defined in the paragraph above Lemma 3.15. Then

$$
a+2 v+1+k_{v}-(a-u)=\left(2 n^{\prime}-1\right)+k_{v}+u .
$$

Since $k_{v}, u \in \mathbb{Z}_{+}$and since $n \leq n^{\prime}$, where $n$ is as defined in the paragraph above Lemma 3.15, we see that

$$
a+2 v+1+k_{v}-(a-u) \geq 2 n^{\prime}-1 \geq 2 n-1 .
$$

By Lemma 2.17 and Remark 2.18 there is a $p$-symmetric arc, $A$, in $C$ with $L_{p}[\bar{x}]=$ $a-u$ for all $[\bar{x}] \in \partial A$ and $L_{p}\left[\bar{\chi}^{A}\right]=a+2 v+1+k_{v}$. This arc will map under $h_{p, g}^{\prime}$ to a $g$-symmetric arc $A^{\prime}$ in $C^{\prime}$ with the property that $L_{g}\left[\bar{x}^{\prime}\right]=b+2$ for all $\left[\bar{x}^{\prime}\right] \in \partial A^{\prime}$ but $L_{g}\left[\bar{\chi}^{A^{\prime}}\right]=b+2 n^{\prime}-1$. This implies that there is a $g$-bridge, $B^{\prime}$, with the property that $L_{g}\left[\bar{\chi}^{B^{\prime}}\right]=2 n^{\prime}-3$ which is a contradiction to Lemma 2.17, Remark 2.18 and the choice of $n^{\prime}$. The lemma follows.

So also in the even case we see that the level $E_{q, 2 u}^{\prime}$ maps to a level that is not below our goal of $E_{p, a+2 u}$. We next show that the level two $q$-points map to a $p$-level of the same parity as $a$.

Lemma 3.17 $h_{q, p}\left(E_{q, 2}^{\prime}\right)=E_{p, a+2 u}$ for some $u \in \mathbb{N}$.

Proof Suppose instead that for some $v \in \mathbb{N} h_{q, p}\left(E_{q, 2}^{\prime}\right)=E_{p, a+2 v+1}$. Then there is a $p$-symmetric $\operatorname{arc} A$ in $C$ with $L_{p}[\bar{x}]=a+1$ and $L_{p}\left[\bar{\chi}^{A}\right]=a+2 v+1$ (this is a shift of a $p$-bridge, $B$, with $L_{p}\left[\bar{\chi}^{B}\right]=2 v$ which we know exists by Lemma 2.16). Let $A^{\prime}=h_{p, g}^{\prime}(A)$ then $L_{g}\left[\bar{x}^{\prime}\right]=b+1$ for all $\left[\bar{x}^{\prime}\right] \in \partial A^{\prime}$ and $L_{g}\left[\bar{\chi}^{A}\right]=b+2$. This implies that there is a $g$-bridge in $B^{\prime} \subset C^{\prime}$, with $L_{g}\left[\bar{\chi}^{B^{\prime}}\right]=1$, which contradicts the fact that $c_{3}^{\prime}=1$.

We now combine the previous lemmas to show that odd levels are mapped to levels of the opposite parity as $a$ and even levels are mapped to levels of the same parity as $a$.

Lemma 3.18 For $d \in \mathbb{N}$, there is $i, j \in \mathbb{Z}_{+}$such that $h_{q, p}\left(E_{q, 2 d}^{\prime}\right)=E_{p, a+2 i}$ and $h_{q, p}\left(E_{q, 2 d+1}^{\prime}\right)=E_{p, a+2 j+1}$. 
Proof We have $h_{q, p}\left(E_{q, 2}^{\prime}\right)=E_{p, a+2 u}$ and $h_{q, p}\left(E_{q, 1}^{\prime}\right)=E_{p, a+1}$. So let $k$ be the least such that $h_{q, p}\left(E_{q, k}^{\prime}\right)=E_{p, a+w}$ and the parity of $k$ is different than the parity of $w$, say $k=2 s$ and $w=2 t+1$ for some $s, t \in \mathbb{Z}_{+}$. Then $h_{q, p}\left(E_{q, k-1}^{\prime}\right)=E_{p, a+2 v+1}$ for some $v \in \mathbb{Z}_{+}$since $k-1$ is odd. Then the difference between $a+2 v+1$ and $a+w$ is even, so there is a $p$-symmetric arc $A$ with $L_{p}[\bar{x}]=a+2 v+1$ for all $[\bar{x}] \in \partial A$ and $L_{p}\left[\bar{\chi}^{A}\right]=a+w$. Then $A^{\prime}=h_{p, g}(A)$ has the property that $L_{g}\left[\bar{x}^{\prime}\right]=b+k-1$ for all $\left[\bar{x}^{\prime}\right] \in \partial A^{\prime}$ and $L_{g}\left[\bar{\chi}^{A^{\prime}}\right]=b+k$. This arc $A^{\prime}$ is then the shift of a $g$-bridge, $B^{\prime}$ with the property that $L_{g}\left[\bar{\chi}^{B^{\prime}}\right]=1$. This contradicts the fact that $c_{3}^{\prime}=1$, ie we have a bridge with center level 1 which can only occur when $c_{3}^{\prime}=0$; see Lemma 2.17 and Remark 2.18.

Finally, we combine all of these lemmas to establish our desired theorem.

Theorem 3.19 Assume $c_{3}^{\prime}=1$ and $c_{3}=1$. Then $h_{q, p}\left(E_{q, j}^{\prime}\right)=E_{p, a+j}$, for all $j \in \mathbb{N}$.

Proof Suppose this is false and let $j$ be the least such that $h_{q, p}\left(E_{q, j}^{\prime}\right) \neq E_{p, a+j}$, say $h_{q, p}\left(E_{q, j}^{\prime}\right)=E_{p, a+j+k}$ for some $k \in \mathbb{N}$. By the previous lemmas we see that $k=2 v$ for some $v \in \mathbb{N}$ (otherwise the parity of $j+k$ would be different from the parity of $j$.)

Enumerate the $p$-levels, $a+j+k+r_{i}$, above or equal to $a+j+k$ that satisfy:

(1) there is a $q$-level that $h_{q, p}$ maps to $p$-level $a+j+k+r_{i}$;

(2) $a+j+k+r_{i}-(a+j-1)$ is odd.

Notice that $r_{1}=0$, because $E_{p+a+j+k}$ satisfies these conditions. Notice that $r_{2}$ will have the property that $h_{q, p}\left(E_{q, j+2}^{\prime}\right)=E_{p, a+j+k+r_{2}}$, and by induction we see that $h_{q, p}\left(E_{q, j+2 i}^{\prime}\right)=E_{p, a+j+k+r_{i}}$.

Choose $r_{i}$ to be the least such that $a+j+k+r_{i}-(a+j-1) \geq 2 n-1$. Then there is a $p$-symmetric arc $A$ with $L_{p}[\bar{x}]=a+j-1$ for all $[\bar{x}] \in \partial A$ and $L_{p}\left[\bar{\chi}^{A}\right]=a+j+k+r_{i}$. Let $A^{\prime}=h_{p, g}^{\prime}(A)$, and notice that $A^{\prime}$ is a $g$-symmetric arc with $L_{g}\left[\bar{x}^{\prime}\right]=b+j-1$ for all $\left[\bar{x}^{\prime}\right] \in \partial A^{\prime}$ and $L_{g}\left[\bar{\chi}^{A^{\prime}}\right]=b+j+2 i$. Then there is a $g$-bridge $B^{\prime}$ with $L_{g}\left[\bar{\chi}^{B^{\prime}}\right]=2 i-1$, and we claim that $2 i-1<2 n^{\prime}-1$. This will establish a contradiction and hence the theorem.

Since $r_{i}$ is minimal, we see that

$$
a+j+k+r_{i-1}-(a+j-1)<2 n-1 \leq a+j+k+r_{i}-(a+j-1) .
$$

So

$$
k+r_{i-1}+1<2 n-1 .
$$


We also know that $r_{i} \geq 2(i-1)$, so combining this with the fact that $k \geq 2$ we see that $k+r_{i-1}+1 \geq 2 i-1$. By the above equation, we get that $2 i-1<2 n-1 \leq 2 n^{\prime}-1$.

Thus in both cases we get that $h_{q, p}$ maps $E_{q, j}^{\prime}$ to $E_{p, a+j}$, for all $j \in \mathbb{N}$, ie, $h_{q, p}\left(E_{q, j}^{\prime}\right)=E_{p+a, j}$, for all $j \in \mathbb{N}$. This implies that $h_{q, p}$ also maps $(q+1)-$ bridges to $(p+a+1)$-bridges. Therefore, for every $j \in \mathbb{N}$, the projection of $E_{q+1, j}^{\prime}$ to the $(q+1)$-st coordinate is above the critical point if, and only if, the projection of $E_{p+a+1, j}$ to the $p+a+1$-st coordinate is above critical point. In other words, $c_{i}^{\prime}=c_{i}$, for all $i \in \mathbb{N}$ and therefore, $s=t$. Thus we have proved:

Main Theorem Let $T_{s}$ and $T_{t}$ be two tent maps with a nonrecurrent critical point and with $\lim _{\longleftarrow}\left\{[0,1], T_{s}\right\}$ homeomorphic to $\lim _{\longleftarrow}\left\{[0,1], T_{t}\right\}$. Then $t=s$.

\section{Appendix: Structure of the composant containing the end- point and quasi-symmetric arcs}

In this appendix we focus on the case that the inverse limit has quasi-symmetric arcs. It is our principle aim to establish Proposition 3.8 mentioned in Section 3.2. We start with the definition of quasi- $p$-symmetric arcs.

Definition 4.1 For $p, n \in \mathbb{Z}_{+}, n>N$, let $\mathcal{C}_{p, n}$ be a chain of $K_{s}$. Let $U$ be an arc of the composant $C$. We say that the arc $U$ is quasi-p-symmetric if $\partial U \subset E_{p}$ and for $U \cap E_{p}=\left\{\left[\bar{x}^{0}\right], \ldots,\left[\bar{x}^{k}\right]\right\}$ we have $L_{p}\left[\bar{x}^{i}\right]=L_{p}\left[\bar{x}^{k-i}\right]$, for every $i=1, \ldots, k-1$, $L_{p}\left[\bar{x}^{0}\right] \neq L_{p}\left[\bar{x}^{k}\right]$ and $\left[\bar{x}^{k}\right] \in \ell_{p, n}^{x^{0}}$, where $\ell_{p, n}^{x^{0}}$ is one of the two links that contain $\left[\bar{x}^{0}\right]$.

That is to say, a quasi- $p$-symmetric arc $A$ is "almost" $p$-symmetric:

(1) $A$ is $p$-symmetric on its interior, but

(2) the boundary points of $A$ have different $p$-levels even though they lie in the same link of $\mathcal{C}_{p, n}$.

Lemma 4.2 If for infinitely many $p \in \mathbb{Z}_{+}$, there are arcs $A_{p} \subset C$ which are quasi$p$-symmetric for $\mathcal{C}_{p, N}$, then for every $q \in \mathbb{Z}_{+}$there is an arc $A_{q} \subset C$ that is quasi-qsymmetric for $\mathcal{C}_{q, N}$.

Proof Let $q \in \mathbb{Z}_{+}$and let $p>q$ be such that $C$ has a quasi- $p$-symmetric arc, $U$, for $\mathcal{C}_{p, N}$. Then $L_{p}\left[\bar{\chi}^{U}\right]=j$ for some $j \in \mathbb{N}$ and if $\partial U=\{[\bar{x}],[\bar{y}]\}$ then $[\bar{x}] \in \ell_{p, N}^{y}$. Clearly $L_{q}\left[\bar{\chi}^{U}\right]=p-q+j=\max \left\{L_{q}[\bar{u}]:[\bar{u}] \in E_{q} \cap \operatorname{Int} U\right\}<\max \left\{L_{q}[\bar{x}], L_{q}[\bar{y}]\right\}$, and, since $\mathcal{C}_{p, N} \prec \mathcal{C}_{q, N}$, we have $[\bar{x}] \in \ell_{q, N}^{y}$. Thus $U$ is quasi-q-symmetric for $\mathcal{C}_{q, N}$ 
Now we provide an example of tent maps and their inverse limit spaces which contain quasi- $p$-symmetric arcs for every $p \in \mathbb{Z}_{+}$.

Example Let $A=a_{1} \cdots a_{\alpha}$ be a finite odd word of length $\alpha$. Let $B$ and $G_{i}, i \in \mathbb{N}$, be finite words of the same parity. Define a sequence of finite words $D_{i}, i \in \mathbb{N}$, inductively by $D_{1}=a_{1} \cdots a_{\alpha-1}\left(1-a_{\alpha}\right) B$ and $D_{i}=D_{i-1} G_{i-1} D_{i-1}$. Also define inductively a sequence of finite words $C_{i}, i \in \mathbb{N}$, by $C_{1}=A B G_{1}$ and $C_{i}=C_{i-1} D_{i-1} G_{i}$. Note that $C_{1}$ is odd. Since $D_{i-1} G_{i}=D_{i-2} G_{i-2} D_{i-2} G_{i}$, and all $G_{i}, i \in \mathbb{N}$, have the same parity, then $D_{i-1} G_{i}$ is even, and hence all $C_{i}, i \in \mathbb{N}$, are odd. Let $A, B$ and $G_{i}, i \in \mathbb{N}$, be such that all $C_{i}, i \in \mathbb{N}$, are $\sigma$-maximal. Let $\gamma_{i}=\left|C_{i}\right|$, for $i \in \mathbb{N}$. Then the sequence $\vec{c}_{1}=\left(c_{i}\right)_{i \in \mathbb{N}}$ such that every initial part of length $\gamma_{i}$ is the finite word $C_{i}$ is a kneading sequence. Note that $\vec{c}_{1}=A B G_{1} D_{1} G_{2} D_{1} G_{1} D_{1} G_{3} D_{1} G_{1} D_{1} G_{2} D_{1} G_{1} D_{1} G_{4} \cdots$ and this kneading sequence is prerecurrent, ie, there exists $k \in \mathbb{N}$ such that $\vec{c}_{k}$ is recurrent.

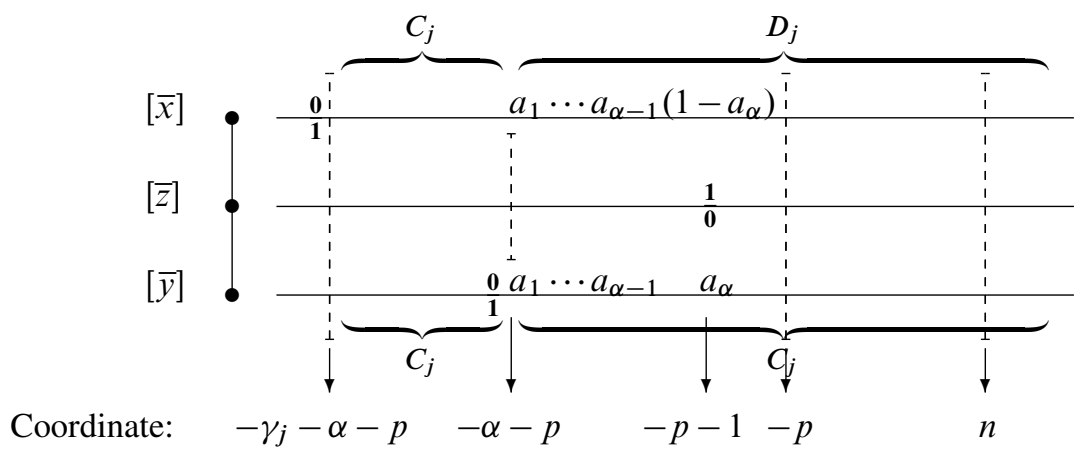

Figure 4: Example

Denote by $K_{s}$ the corresponding continuum. For $p, n \in \mathbb{Z}_{+}$, let $\mathcal{C}_{p, n}$ be a chain of $K_{s}$. Let $j \in \mathbb{N}$ be such that $\left|D_{j}\right|>p+n+\alpha$. Let $\bar{x}=\overleftarrow{0^{\infty}} \vec{c}_{1}$ be such that $L_{p}[\bar{x}]=\gamma_{j}+\alpha+1$. Let $\bar{y}=\overleftarrow{0}^{\infty} C_{j} \vec{c}_{1}$ be such that $L_{p}[\bar{y}]=\alpha+1$. Since $\vec{x}_{-p-\gamma_{j}-\alpha-1}=C_{j} D_{j} \cdots$ and $\vec{y}_{-p-\gamma_{j}-\alpha-1}=C_{j} C_{j} \cdots$, then $x_{i}=y_{i}$, for every $i \leq n$, $i \neq-p-1$, and $x_{-p-1} \neq y_{-p-1}$; see Figure 4. Therefore, the arc $U$ between the points $[\bar{x}]$ and $[\bar{y}]$ contains only one $p$-point, $[\bar{z}]$, and $L_{p}[\bar{z}]=1$. Also, $[\bar{y}] \in \ell_{p, n}^{x}$ and the $\operatorname{arc} U$ is quasi- $p$-symmetric with respect to $\mathcal{C}_{p, n}$.

Remark 4.3 Notice that if $\vec{c}_{1}$ is not prerecurrent ie, if the corresponding inverse limit $K_{S}$ contains quasi- $p$-symmetric arcs for finitely many $p \in \mathbb{Z}_{+}$, then there is 
some $P \in \mathbb{Z}_{+}$such that, for $p \geq P, C$ does not have any quasi- $p$-symmetric arcs for $\mathcal{C}_{p, N}$. Then $C$ does not have any quasi-0-symmetric arcs for $\mathcal{C}_{0, N+P}$ and thus no quasi- $p$-symmetric arcs for all $p \in \mathbb{Z}_{+}$and $n \geq N+P$. Let $S_{1}=N+P$. Then, in the case that $\vec{c}_{1}$ is not prerecurrent, for all $p \in \mathbb{Z}_{+}$and $n \geq S_{1}, C$ does not have any quasi- $p$-symmetric arcs for $\mathcal{C}_{p, n}$.

In the next two lemmas we give a comparison of the $p$-levels of the boundary points of a quasi- $p$-symmetric arc and its center. The next lemma is a consequence of the nonrecurrence of $T(1 / 2)$.

Lemma 4.4 Let $p, n \in \mathbb{Z}_{+}$and $n>N$. Let $U$ be a quasi-p-symmetric arc of the composant $C$ with respect to $\mathcal{C}_{p, n}$. Let $U \cap E_{p}=\left\{\left[\bar{x}^{0}\right], \ldots,\left[\bar{x}^{k}\right]\right\}$ and let $l_{i}=L_{p}\left[\bar{x}^{i}\right]$. Then for $l=\min \left\{l_{0}, l_{k}\right\}$ we have $l_{k / 2}<l$ and $l-l_{k / 2} \leq R$.

Proof Without loss of generality we can assume that $l_{0}>l_{k}$. Therefore, $l_{k / 2}<l_{0}$ $\left(l_{k / 2}>l_{0}\right.$ would imply $\left.l_{0}=l_{k}\right)$. So we have either $l_{0}>l_{k / 2}>l_{k}$ or $l_{k / 2}<\min \left\{l_{0}, l_{k}\right\}$. Assume, in order to achieve a contradiction, that $l_{0}>l_{k / 2}>l_{k}$. Since $\left[\bar{x}^{0}\right] \in \ell_{p, n}^{x^{k}}$, we have

$$
x_{-p}^{0} \cdots x_{n-\rho}^{0}=x_{-p}^{k} \cdots x_{n-\rho}^{k},
$$

for some $\rho<R$. Since the arc between $\left[\bar{x}^{1}\right]$ and $\left[\bar{x}^{k-1}\right]$ is $p$-symmetric, ie, the number of $p$-points of $p$-level $l_{i}, i \neq k / 2$, in Int $U$ is even, then by Remark 2.12 we have

$$
x_{-p-l_{k}+1}^{0} \cdots x_{-p-1}^{0}=x_{-p-l_{k}-1}^{k} \cdots x_{-p-1}^{k} .
$$

Now (2) and (3) imply

$$
x_{-p-l_{k}+1}^{0} \cdots x_{n-\rho}^{0}=x_{-p-l_{k}+1}^{k} \cdots x_{n-\rho}^{k} .
$$

On the other hand, $L_{p}\left[\bar{x}^{0}\right]=l_{0}$ implies

$$
x_{-p-l_{k}+1}^{0} \cdots x_{n-\rho}^{0}=c_{l_{0}-l_{k}+1} \cdots c_{p+l_{0}+n-\rho}
$$

and $L_{p}\left[\bar{x}^{k}\right]=l_{k}$ implies

$$
x_{-p-l_{k}+1}^{k} \cdots x_{n-\rho}^{k}=c_{1} \cdots c_{p+l_{k}+n-\rho} .
$$

Now (4) and (5) imply

$$
c_{l_{0}-l_{k}+1} \cdots c_{p+l_{0}+n-\rho}=c_{1} \cdots c_{p+l_{k}+n-\rho} .
$$


Since $p+l_{k}+n-\rho>n-\rho>n-R>R$, this contradicts the assumption that the critical point is not recurrent. Therefore, $l_{k / 2}<\min \left\{l_{0}, l_{k}\right\}$. Since the arc between $\left[\bar{x}^{1}\right]$ and $\left[\bar{x}^{k-1}\right]$ is $p$-symmetric, then $l_{k / 2}=\max \left\{l_{1}, \ldots, l_{k-1}\right\}$ and

$$
\begin{aligned}
c_{1} \cdots c_{l_{k}-l_{k / 2}-1} & =x_{-p-l_{k}+1}^{k} \cdots x_{-p-l_{k / 2}-1}^{k} \\
& =x_{-p-l_{k}+1}^{0} \cdots x_{-p-l_{k / 2}-1}^{0}=c_{l_{0}-l_{k}+1} \cdots c_{l_{0}-l_{k / 2}-1} .
\end{aligned}
$$

Since the critical point is not recurrent, then $l_{k}-l_{k / 2} \leq R$.

We use the nonrecurrence of $T(1 / 2)$ and $T^{2}(1 / 2)$ to prove that the level of the center of a quasi- $p$-symmetric arc is at least two lower than the levels of the boundary points.

Lemma 4.5 Let $p, n \in \mathbb{Z}_{+}$and $n>N$. Let $A$ be a quasi-p-symmetric arc for $\mathcal{C}_{p, n}$ with $A \cap E_{p}=\left\{\left[\bar{x}^{0}\right], \ldots,\left[\bar{x}^{k}\right]\right\}$. Then

$$
L_{p}\left[\bar{x}^{k / 2}\right]<\min \left\{L_{p}\left[\bar{x}^{0}\right]-1, L_{p}\left[\bar{x}^{k}\right]-1\right\} .
$$

Proof Let $l_{i}=L_{p}\left[\bar{x}^{i}\right]$, and suppose that $l_{k / 2}=l_{0}-1$. Let $\left[\bar{z}^{0}\right]=\tilde{\sigma}^{-l_{k / 2}+1}\left[\bar{x}^{0}\right]$ and $\left[\bar{z}^{1}\right]=\tilde{\sigma}^{-l_{k / 2}+1}\left[\bar{x}^{k}\right]$. Since there is $\ell_{p, n}^{x^{0}}$ such that $\left[\bar{x}^{k}\right] \in \ell_{p, n}^{x^{0}}$, then either

$$
x_{-p-k / 2+1}^{0} \cdots x_{n}^{0}=x_{-p-k / 2+1}^{k} \cdots x_{n}^{k},
$$

or there is $\rho$ such that

$$
\begin{aligned}
x_{-p-k / 2+1}^{0} \cdots x_{n-\rho-1}^{0} & =x_{-p-k / 2+1}^{k} \cdots x_{n-\rho-1}^{k}, \\
x_{n-\rho}^{0} & \neq x_{n-\rho}^{k},
\end{aligned}
$$

and

$$
x_{n-\rho+1}^{0} \cdots x_{n}^{0}=x_{n-\rho+1}^{k} \cdots x_{n}^{k}=c_{1} \cdots c_{\rho} \text {. }
$$

Therefore, either

or

$$
z_{-p}^{0} \cdots z_{n+k / 2-1}^{0}=z_{-p}^{1} \cdots z_{n+k / 2-1}^{1},
$$

$$
z_{-p}^{0} \cdots z_{n+k / 2-\rho-2}^{0}=z_{-p}^{1} \cdots z_{n+k / 2-\rho-2}^{1},
$$

$$
z_{n+k / 2-\rho-1}^{0} \neq z_{n+k / 2-\rho-1}^{1},
$$

and

$$
z_{n+k / 2-\rho}^{0} \cdots z_{n+k / 2-1}^{0}=z_{n+k / 2-\rho}^{1} \cdots z_{n+k / 2-1}^{1}=c_{1} \cdots c_{\rho} .
$$

Since $\rho<R$, then $\rho-k / 2<R$. This implies that there exists $\ell_{p, n}^{z^{0}}$ such that $\left[\bar{z}^{1}\right] \in \ell_{p, n}^{z^{0}}$. By our assumption that $l_{k / 2}=l_{0}-1$ we see that $L_{p}\left[\bar{z}^{0}\right]=2$. So $\vec{z}_{-p}^{0}=\vec{c}_{2}$. Since $n$ was chosen, among other things, to ensure that $p$-points which project to $\vec{c}_{2}$ in the $p$-th coordinate cannot be in a link with any $p$-points with different levels (Remark 2.23), we have a contradiction because $L_{p}\left[\bar{z}^{1}\right] \neq 2$. 
In the next lemma we prove that the number of adjacent quasi- $p$-symmetric arcs is bounded. This is a consequence of Theorem 2.19 which says that the number of $p$-points in a $p$-bridge is bounded.

Lemma 4.6 Let $p, n \in \mathbb{Z}_{+}$and $n>N$. Then there exists an $L \in \mathbb{N}$ such that if $A$ and $D$ are arcs of the composant $C$ with the following properties:

(1) $A$ is quasi-p-symmetric with respect to $\mathcal{C}_{p, n}, A \cap E_{p}=\left\{\left[\bar{x}^{0}\right], \ldots,\left[\bar{x}^{k}\right]\right\}$, and $A$ does not properly contain any quasi- $p$-symmetric arcs.

(2) $D \cap E_{p}=\left\{\left[\bar{z}^{0}\right], \ldots,\left[\bar{z}^{d}\right]\right\}$ and there exists $j \in\{0, \ldots, d-k\}$, such that $\left[\bar{z}^{j+i}\right]=$ $\left[\bar{x}^{i}\right]$, for $i=0, \ldots, k$, ie, the arc between $\left[\bar{z}^{j}\right]$ and $\left[\bar{z}^{j+k}\right]$ is $A$.

(3) $\left[\bar{z}^{j+l k}\right] \in \ell_{p, n}^{x^{0}}$, for $l \in \mathbb{Z}$ such that $0 \leq j+l k \leq d$ and for some $\ell_{p, n}^{x^{0}}$.

(4) $L_{p}\left[\bar{z}^{j+l k+i}\right]=L_{p}\left[\bar{x}^{i}\right]$, for $i \in\{1, \ldots, k-1\}$ and $l \in \mathbb{Z}$ such that $0 \leq j+l k+i \leq d$.

Then $d / k \leq L$.

Note that (3) and (4) imply that the arc $A_{l}$ between $\left[\bar{z}^{j+l k}\right]$ and $\left[\bar{z}^{j+(l+1) k}\right]$ is quasi- $p$-symmetric and $\operatorname{FP}\left(\operatorname{Int} A_{l}\right)=\operatorname{FP}(\operatorname{Int} A)$. Also $d / k$ is the number of quasi$p$-symmetric arcs $A_{l}$ contained in $D$.

Proof Since $A$ is quasi- $p$-symmetric, then $l_{k / 2}>L_{p}\left[\bar{x}^{i}\right]$, for every $i \in\left\{1, \ldots, \frac{k}{2}-1\right\}$ and $l_{k / 2}<\min \left\{L_{p}\left[\bar{x}^{0}\right]-1, L_{p}\left[\bar{x}^{k}\right]-1\right\}$. Therefore, $\widetilde{\sigma}^{-l_{k / 2}-1}(A)$ is contained in a $p$-bridge, ie, $\tilde{\sigma}^{-l_{k / 2}-1}(A)$ doesn't contain any $p$-point with zero $p$-level. Since $D$ satisfies (3) and (4), $\tilde{\sigma}^{-l_{k / 2}-1}(D)$ is contained in the same $p$-bridge as $\widetilde{\sigma}^{-l_{k / 2}-1}(A)$. Denote this $p$-bridge by $B$. Note that $\left[\bar{y}^{l}\right]=\widetilde{\sigma}^{-l_{k / 2}-1}\left(\left[\bar{z}^{j+l k}\right]\right) \in B$, for every $l \in \mathbb{Z}$ with $0 \leq j+l k \leq d$. But the number of $p$-points of a $p$-bridge is limited by Theorem 2.19. Let us denote by $L$ the largest number of $p$-points of a $p$-bridge. Although the arc $D$ contains more $p$-points then the arc $\tilde{\sigma}^{-l_{k / 2}-1}(D)$, the number $d / k$ of $p$-points of $D$ contained in links which contain $\left[\bar{x}^{0}\right]$ is less than or equal $L$.

Remark 4.7 Note that, by Theorem 2.19, $L_{p}\left[\bar{y}^{l}\right]<M$, for all $l \in \mathbb{Z}$ with $0 \leq j+l k \leq$ $d$ and $\left[\bar{y}^{l}\right] \neq\left[\bar{\chi}^{B}\right]$, where $\left[\bar{y}^{l}\right]=\tilde{\sigma}^{-l_{k / 2}-1}\left(\left[\bar{z}^{j+l k}\right]\right)$, as in the proof of Lemma 4.6. Therefore, there exists an $S_{2} \geq N$ such that for all arcs $A$ and $D$ which satisfy (1)-(4) of Lemma 4.6, the following holds: There are at most two points $\left[\bar{z}^{a}\right],\left[\bar{z}^{b}\right] \in D \cap E_{p}$ such that the arc between them is quasi- $p$-symmetric with respect to $\mathcal{C}_{p, S_{2}}$. Since for all $n \geq S_{2}, \mathcal{C}_{p, n} \prec \mathcal{C}_{p, S_{2}}$ this property holds for all larger $n$.

Note also that $\left[\bar{z}^{a}\right]$ and $\left[\bar{z}^{b}\right]$ have the following property: For one of them, let's say $\left[\bar{z}^{a}\right]$, we have $\left[\bar{z}^{a}\right]=\tilde{\sigma}^{l_{k / 2}+1}\left(\left[\bar{\chi}^{B}\right]\right)$, and for the other one, let's say $\left[\bar{z}^{b}\right]$, there exists $l$ 
such that $\left[\bar{z}^{b}\right]=\widetilde{\sigma}^{l_{k / 2}+1}\left(\left[\bar{y}^{l}\right]\right)$ and $L_{p}\left[\bar{y}^{l}\right]<M$. Therefore, there exists some $S_{3} \geq S_{2}$ large enough so that whenever two points, $[\bar{u}]$ and $[\bar{v}]$, are boundary points of a quasi$p$-symmetric arc, then $\left|L_{p}[\bar{u}]-L_{p}[\bar{v}]\right|>M$.

Let $S=\max \left\{S_{1}, S_{2}, S_{3}\right\}$, where $S_{1}$ is defined in Remark 4.3 for $\vec{c}_{1}$ which is not prerecurrent.

Recall that the main result of this Appendix, Proposition 3.8, addresses the $h_{q, p}$-image of a quasi-q-symmetric arc. One thing which is apparent about quasi-q-symmetric arcs is that they are $q$-link-symmetric, and so it is simple to see that their images are $p$-link-symmetric. So now we turn our attention to properties of $p$-link-symmetric arcs.

In the next lemma we prove that every $p$-link-symmetric arc $A$ is "almost" $p-$ symmetric, ie, there exists at most one link which contains a pair of $p$-points of $A$ that have different $p$-levels.

Lemma 4.8 Let $p, n \in \mathbb{Z}_{+}$and $n>S$. Let $A$ be an arc such that $A \cap E_{p}=$ $\left\{\left[\bar{y}^{0}\right], \ldots,\left[\bar{y}^{j}\right], \ldots,\left[\bar{y}^{2 j}\right]\right\}$ and $\left[\bar{y}^{j-i}\right] \in \ell_{p, n}^{y^{j+i}}$ for all $i \in\{1, \ldots, j\}$. Then there is at most one $k \leq j$ such that $L_{p}\left[\bar{y}^{j-k}\right] \neq L_{p}\left[\bar{y}^{j+k}\right]$.

Proof For each $i$ let $l_{i}=L_{p}\left[\bar{y}^{i}\right]$. By Lemma 4.4 if $l_{j-1}<l_{j}$ then $l_{j+1}=l_{j-1}$. Choose $k \leq j$. If $l_{j-i}<l_{j}$ for all $i<k$ then by the same lemma we get that $l_{j-i}=l_{j+i}$. Suppose that $k$ is minimal such that $l_{j-k}>l_{j}$. Then we also have that $l_{j+k}>l_{j}$. So we have that for all $i<k, l_{j-i}=l_{j+i}<l_{j}$, and $\left[\bar{y}^{j-k}\right] \in \ell_{p, n}^{y^{j+k}}$. It is easy to see that $l_{j-k} \neq l_{j+k}$. Let $G$ be the arc with $\partial G=\left\{\left[\bar{y}^{j-k}\right],\left[\bar{y}^{j+k}\right]\right\}$. Then $G$ is quasi- $p$-symmetric.

Assume without loss of generality that $l_{j+k}>l_{j-k}$. Then, $l_{j+k}-l_{j}-1 \geq M$, by Remark 4.7. Hence $\tilde{\sigma}^{-l_{j}-1}\left[\bar{y}^{j+k}\right]$ is the center of some $p$-bridge, $B$, containing $\tilde{\sigma}^{-l_{j}-1}\left[\bar{y}^{j-k}\right]$. Since $B$ is $p-$ symmetric around its center $\tilde{\sigma}^{-l_{j}-1}\left[\bar{y}^{j+k}\right]$, there is a $p$-symmetric arc $H$ with $\partial H=\left\{\widetilde{\sigma}^{-l_{j}-1}\left[\bar{y}^{j-k}\right], \widetilde{\sigma}^{-l_{j}-1}\left[\bar{y}^{j+3 k}\right]\right\}$. Then consider $\tilde{\sigma}^{l+1}(H)=H^{\prime}$. This $H^{\prime}$ is a $p$-symmetric arc with $\partial H^{\prime}=\left\{\left[\bar{y}^{j-k}\right],\left[\bar{y}^{j+3 k}\right]\right\}$ and with center $\left[\bar{y}^{j+k}\right]$. This implies that $l_{j-k}=l_{j+3 k}, l_{j}=l_{j+2 k}$, and $l_{i} \leq l_{j+k}$ for all $i, j-k \leq i \leq j+3 k$. Thus we have:

(1) for all $i, 1 \leq i \leq k, l_{j-i}=l_{j+i}<l_{j}$ and

(2) for all $i, 1 \leq i \leq 2 k, l_{j+k-i}=l_{j+k+i}$. 
Hence for all $i$ such that $j-k<i<j+k$ or $j+k<i<j+3 k$ we have $l_{i} \leq l_{j}$. Suppose there is a $k_{2}>k$ such that $l_{j-k_{2}}>l_{j}$ and $\left[\bar{y}^{j-i}\right] \in \ell_{p, n}^{y^{j+i}}$ for all $i \in\left\{0, \ldots, k_{2}\right\}$. Suppose further that $k_{2}<3 k$. Then $\left[\bar{y}^{j+k_{2}}\right] \in \widetilde{\sigma}^{l_{j}+1}(H)$ which is a symmetric arc with center of symmetry $\left[\bar{y}^{j+k}\right]$. Then $l_{j+k_{2}} \leq l_{j}$ and hence $l_{j-k_{2}}=l_{j+k_{2}}<l_{j}$.

Next suppose that $k_{2} \geq 3 k$. Then $\left[\bar{y}^{j-3 k}\right] \in \ell_{p, n}^{y^{j+3 k}}$, and $l_{j-k}=l_{j+3 k}$. So $\left[\bar{y}^{j-k}\right] \in$ $\ell_{p, n}^{y^{j+3 k}}$ and $\left[\bar{y}^{j+k}\right] \in \ell_{p, n}^{y^{j+3 k}}$. Since $n \geq S$ we have either $l_{j-3 k}=l_{j-k}$ or $l_{j-3 k}=l_{j+k}$. Either way we get a contradiction because this will lead to an arc with boundary points with the same $p$-level but all of the interior $p$-points will have lower level.

In the next lemma we show that symmetry around the boundary point of a quasi- $p-$ symmetric arc with lower level cannot be extended "too far".

Lemma 4.9 Let $p, n \in \mathbb{Z}_{+}$and $n>S$. Let $A$ be a quasi-p-symmetric arc with $A \cap E_{p}=\left\{\left[\bar{x}^{0}\right], \ldots,\left[\bar{x}^{k}\right]\right\}$ and $L_{p}\left[\bar{x}^{0}\right]>L_{p}\left[\bar{x}^{k}\right]$. Let $j$ be the largest number such that $L_{p}\left[\bar{x}^{k+i}\right]=L_{p}\left[\bar{x}^{k-i}\right]$ for all $i \in\{0, \ldots, j\}$. Then $0 \leq j<k$.

Proof Since $L_{p}\left[\bar{x}^{0}\right]>L_{p}\left[\bar{x}^{k}\right]$, then $L_{p}\left[\bar{x}^{0}\right]>L_{p}\left[\bar{x}^{i}\right]$ for all $i \in\{1, \ldots, k\}$. Assume by contradiction that $j \geq k$. Then the arc $D$ between $\left[\bar{x}^{0}\right]$ and $\left[\bar{x}^{2 k}\right]$ is $p$-symmetric and for its center of symmetry $\left[\bar{x}^{k}\right]$ we have $L_{p}\left[\bar{x}^{k}\right]<L_{p}\left[\bar{x}^{0}\right]=L_{p}\left[\bar{x}^{2 k}\right]$, a contradiction. Therefore, $L_{p}\left[\bar{x}^{k+i}\right]=L_{p}\left[\bar{x}^{k-i}\right]$ for all $i \in\{0, \ldots, j\}$ implies $j<k$.

Finally we prove Proposition 3.8 that for every quasi-q-symmetric arc $A^{\prime}$ of $C^{\prime}$, the $\operatorname{arc} h_{q, p}\left(A^{\prime}\right)$ is not $p$-symmetric.

Proof of Proposition 3.8 Let $A^{\prime}$ be a quasi-q-symmetric arc, and let $A^{\prime} \cap E_{q}^{\prime}=$ $\left\{\left[\bar{x}^{\prime 0}\right], \ldots,\left[\bar{x}^{\prime k}\right]\right\}$. Let $A=h_{q, p}\left(A^{\prime}\right)$, and let $A \cap E_{p}=\left\{\left[\bar{y}^{0}\right], \ldots,\left[\bar{y}^{l}\right]\right\}$. We want to prove that the $\operatorname{arc} h_{q, p}\left(A^{\prime}\right)$ is not $p$-symmetric, or more precisely that there exists an $r, 0 \leq r \leq l$, such that $L_{p}\left[\bar{y}^{r}\right] \neq L_{p}\left[\bar{y}^{l-r}\right]$.

Without loss of generality we can assume that $L_{q}\left[\bar{x}^{\prime 0}\right]>L_{q}\left[\bar{x}^{\prime k}\right]$. Let $j$ be the largest number such that $L_{q}\left[\bar{x}^{\prime k+i}\right]=L_{q}\left[\bar{x}^{\prime k-i}\right]$, for all $i \in\{0, \ldots, j\}$, and $L_{q}\left[\bar{x}^{\prime k+j+1}\right] \neq$ $L_{q}\left[\bar{x}^{\prime k-j-1}\right]$. Then, by Lemma 4.9 , we have $0 \leq j<k$.

(1) Suppose that $k / 2 \leq j<k$. Assume by contradiction that $A^{\prime}$ is mapped to a $p-$ symmetric arc, ie, $L_{p}\left[\bar{y}^{i}\right]=L_{p}\left[\bar{y}^{l-i}\right]$ for $i \in\{0, \ldots, l\}$. Since $L_{q}\left[\bar{x}^{\prime k-i}\right]=L_{q}\left[\bar{x}^{\prime k+i}\right]$ for $i \in\{1, \ldots, j\}$, then by Lemma 3.6 we have $\left[\bar{y}^{l+i}\right] \in \ell_{p, n}^{y^{l-i}}$ for all $i \in\{1, \ldots, a\}$, where $a$ is such that $h_{q, p}\left(\left[\bar{x}^{\prime k+j}\right]\right)=\left[\bar{y}^{l+a}\right]$. Obviously we have $l / 2 \leq a<l$; see Figure 5. Since $A$ is $p$-symmetric, $L_{p}\left[\bar{y}^{l / 2}\right]>L_{p}\left[\bar{y}^{l}\right]$ and hence $L_{p}\left[\bar{y}^{l / 2}\right] \neq$ $L_{p}\left[\bar{y}^{3 l / 2}\right]$. This fact together with Lemma 4.8 implies that $L_{p}\left[\bar{y}^{l+i}\right]=L_{p}\left[\bar{y}^{l-i}\right]$ 

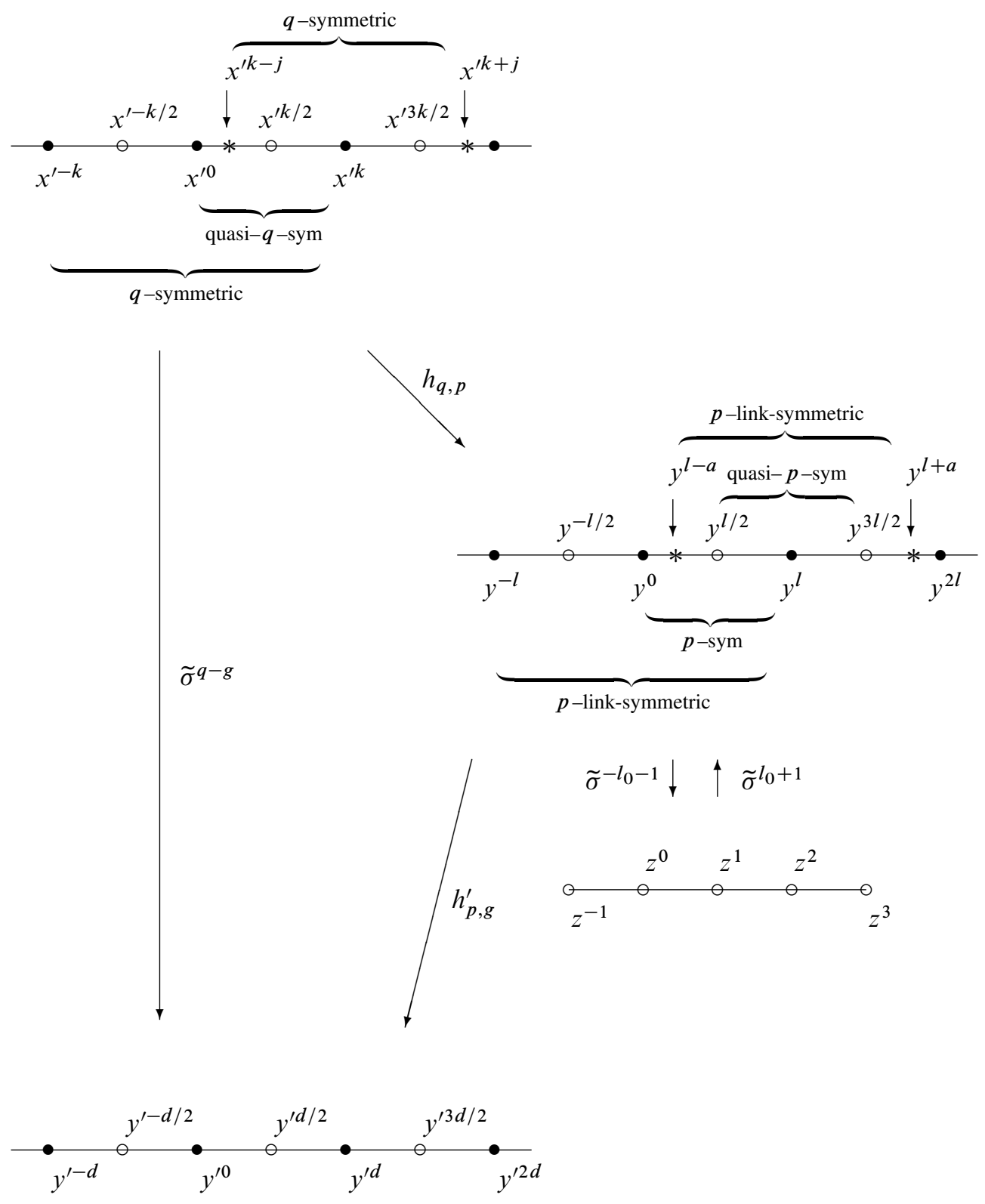

Figure 5: The configuration in part (1) of the proof of Proposition 3.8 
for all $i \in\{1, \ldots, a\}, i \neq l / 2$. Therefore, the arc between $\left[\bar{y}^{l / 2}\right]$ and $\left[\bar{y}^{3 l / 2}\right]$ is quasi- $p$-symmetric.

By Remark 4.7 $L_{q}\left[\bar{x}^{\prime 0}\right]-L_{q}\left[\bar{x}^{\prime k}\right]>M$ and hence $L_{q}\left[\bar{x}^{\prime-i}\right]=L_{q}\left[\bar{x}^{\prime i}\right]$ for $i \in\{1, \ldots, k\}$. Therefore, by Lemma 3.6 we have $\left[\bar{y}^{-i}\right] \in \ell_{p, n}^{y^{i}}$ for $i \in\{1, \ldots, l\}$. Again, since $L_{p}\left[\bar{y}^{l / 2}\right] \neq L_{p}\left[\bar{y}^{-l / 2}\right]$, Lemma 4.8 implies that $L_{p}\left[\bar{y}^{-i}\right]=L_{p}\left[\bar{y}^{i}\right]$ for $i \in\{1, \ldots, l\}$, $i \neq l / 2$. Therefore, the arc between $\left[\bar{y}^{-l / 2}\right]$ and $\left[\bar{y}^{l / 2}\right]$ is also quasi-p-symmetric and by Remark 4.7 $L_{p}\left[\bar{y}^{l / 2}\right]>L_{p}\left[\bar{y}^{-l / 2}\right]=L_{p}\left[\bar{y}^{3 l / 2}\right]$.

Let $l_{i}=L_{p}\left[\bar{y}^{i}\right]$. Let $D$ be the arc from $\left[\bar{y}^{-l / 2}\right]$ to $\left[\bar{y}^{3 l / 2}\right]$. Consider the $\operatorname{arc} \widetilde{\sigma}^{-l_{0}-1}(D)$. Let $\left[\bar{z}^{0}\right]=\widetilde{\sigma}^{-l_{0}-1}\left[\bar{y}^{-l / 2}\right],\left[\bar{z}^{1}\right]=\widetilde{\sigma}^{-l_{0}-1}\left[\bar{y}^{l / 2}\right]$ and $\left[\bar{z}^{2}\right]=\widetilde{\sigma}^{-l_{0}-1}\left[\bar{y}^{3 l / 2}\right]$. The folding pattern of $\tilde{\sigma}^{-l_{0}-1}(D)$ is

$$
l_{-l / 2}-l_{0}-1 l_{l / 2}-l_{0}-1 \quad l_{3 l / 2}-l_{0}-1 .
$$

Let $\left[\bar{z}^{-1}\right]$ be the $p$-point immediately before $\left[\bar{z}^{0}\right]$ and let $\left[\bar{z}^{3}\right]$ be the $p$-point immediately after $\left[\bar{z}^{2}\right]$. By Lemma 4.5 we have $l_{-l / 2}-l_{0}-1 \neq 0 \neq l_{3 l / 2}-l_{0}-1$. Therefore, $\left[\bar{z}^{-1}\right],\left[\bar{z}^{0}\right],\left[\bar{z}^{1}\right],\left[\bar{z}^{2}\right],\left[\bar{z}^{3}\right]$ all belong to the same $p$-bridge, say $B$. Since the arc between $\left[\bar{y}^{-l / 2}\right]$ and $\left[\bar{y}^{l / 2}\right]$ is quasi- $p$-symmetric, then by Remark 4.7 we have $l_{l / 2}-l_{0}>l_{l / 2}-l_{-l / 2}>M$. This implies $L_{p}\left(z^{1}\right)=l_{l / 2}-l_{0}-1 \geq M$ and by Theorem $2.19,\left[\bar{z}^{1}\right]$ is the center of $\hat{B}$, ie, the arc $B$ between $\left[\bar{z}^{-1}\right]$ to $\left[\bar{z}^{3}\right]$ is $p$-symmetric; see Figure 5. Then $\widetilde{\sigma}^{l_{0}+1}(B)$ is $p$-symmetric with center $\widetilde{\sigma}^{l_{0}+1}\left[\bar{z}^{1}\right]=\left[\bar{y}^{l / 2}\right]$. Since $l_{-l}=l_{0}$, then $\left[\bar{y}^{-l}\right] \in \widetilde{\sigma}^{l_{0}+1}(B)$. Therefore, $\widetilde{\sigma}^{l_{0}+1}(B)$ contains $\left[\bar{y}^{2 l}\right]$ and $L_{p}\left[\bar{y}^{2 l}\right]=$ $L_{p}\left[\bar{y}^{l}\right]=L_{p}\left[\bar{y}^{0}\right]=L_{p}\left[\bar{y}^{-l}\right]$.

Now let $G$ be the arc from $\left[\bar{y}^{-l}\right]$ to $\left[\bar{y}^{2 l}\right]$. The arc $G$ is $p$-symmetric with center of symmetry $\left[\bar{y}^{l / 2}\right]$. Let $G^{\prime}=h_{p, g}^{\prime}(G)$, and let $G^{\prime} \cap E_{g}^{\prime}=\left\{\left[\bar{y}^{\prime-d}\right], \ldots,\left[\bar{y}^{\prime 2 d}\right]\right\}$. By construction and by Lemma 3.6 we see that $\left[\bar{y}^{\prime-d}\right]=\left[\bar{x}^{\prime-k}\right],\left[\bar{y}^{\prime-d / 2}\right]=\left[\bar{x}^{\prime-k / 2}\right]$, $\left[\bar{y}^{\prime 0}\right]=\left[\bar{x}^{\prime 0}\right],\left[\bar{y}^{\prime d / 2}\right]=\left[\bar{x}^{\prime k / 2}\right],\left[\bar{y}^{\prime d}\right]=\left[\bar{x}^{\prime k}\right],\left[\bar{y}^{\prime 3 d / 2}\right]=\left[\bar{x}^{\prime 3 k / 2}\right]$, and $\left[\bar{y}^{\prime 2 d}\right]=\left[\bar{x}^{\prime 2 k}\right]$. Since $G$ is $p$-symmetric, by Lemma 3.6 we have $\left[\bar{y}^{\prime d / 2+i}\right] \in \ell_{g, e}^{y^{\prime d / 2-i}}$ for all $i \in$ $\{1, \ldots, 3 d / 2\}$. Since $L_{q}\left[\bar{x}^{\prime 0}\right] \neq L_{q}\left[\bar{x}^{\prime k}\right]$, then $L_{g}\left[\bar{y}^{\prime 0}\right] \neq L_{g}\left[\bar{y}^{\prime d}\right]$, and this fact together with Lemma 4.8 implies that $L_{g}\left[\bar{y}^{\prime d / 2-i}\right]=L_{g}\left[\bar{y}^{\prime / 2+i}\right]$ for $i \in\{1, \ldots, 3 d / 2\}, i \neq$ $d / 2$. Particularly, $L_{g}\left[\bar{y}^{\prime d+i}\right]=L_{g}\left[\bar{y}^{\prime-i}\right]$, for every $i \in\{1, \ldots, d\}$. Since the arc between $\left[\bar{x}^{\prime-k}\right]$ and $\left[\bar{x}^{\prime k}\right]$ is $q$-symmetric, then the arc between $\left[\bar{y}^{\prime-d}\right]$ and $\left[\bar{y}^{\prime d}\right]$ is $g$-symmetric. Since the arc between $\left[\bar{x}^{\prime 0}\right]$ and $\left[\bar{x}^{\prime k}\right]$ is quasi-q-symmetric, then the arc between $\left[\bar{y}^{\prime 0}\right]$ and $\left[\bar{y}^{\prime d}\right]$ is quasi-g-symmetric Therefore, $L_{g}\left[\bar{y}^{\prime d+i}\right]=L_{g}\left[\bar{y}^{\prime d-i}\right]$, for every $i \in\{1, \ldots, d-1\}$ and every $g$-point between $\left[\bar{y}^{\prime}\right]$ and $\left[\bar{y}^{\prime 2 d}\right]$ has a lower $g$-level than $\left[\bar{y}^{\prime d}\right]$. But $L_{g}\left[\bar{y}^{\prime d}\right]=L_{g}\left[\bar{y}^{\prime 2 d}\right]$, a contradiction.

(2) Now suppose instead that $j<k / 2$. Again we suppose for contradiction that $A^{\prime}$ is mapped to a $p$-symmetric arc $A$, ie, $L_{p}\left[\bar{y}^{i}\right]=L_{p}\left[\bar{y}^{l-i}\right]$ for $i \in\{0, \ldots, l\}$. Since $j<k / 2$, there is some $a<l / 2$ with $h_{q, p}\left[\bar{x}^{\prime k+j}\right]=\left[\bar{y}^{l+a}\right]$ and $\left[\bar{y}^{l+i}\right] \in \ell_{p, n}^{y^{l-i}}$ for 
$i \in\{1, \ldots, a\}$. Since $L_{q}\left[\bar{x}^{\prime-i}\right]=L_{q}\left[\bar{x}^{\prime i}\right]$ for all $i \in\{1, \ldots, k\}$ we see that $\left[\bar{y}^{-i}\right] \in \ell_{p, n}^{y^{i}}$ for all $i \in\{1, \ldots, l\}$, and by Lemma 4.8 this implies that $L_{p}\left[\bar{y}^{-i}\right]=L_{p}\left[\bar{y}^{i}\right]$ for all $i \in\{1, \ldots, l\}$ except for $i=l / 2$. So the arc from $\left[\bar{y}^{-l / 2}\right]$ to $\left[\bar{y}^{l / 2}\right]$ is quasi-psymmetric. Since $\left[\bar{y}^{l+i}\right] \in \ell_{p, n}^{y^{l-i}}$ for $i \in\{1, \ldots, a\}$, and $L_{p}\left[\bar{y}^{l-i}\right]<L_{p}\left[\bar{y}^{l}\right]$ for every $i \in\{1, \ldots, l-1\}$, by Lemma 4.8 and Lemma 4.4 we see that $L_{p}\left[\bar{y}^{l+i}\right]=L_{p}\left[\bar{y}^{l-i}\right]$ for $i \in\{1, \ldots, a\}$. Now we have two subcases:

(a) $L_{p}\left[\bar{y}^{l / 2}\right]>L_{p}\left[\bar{y}^{-l / 2}\right]$,

(b) $L_{p}\left[\bar{y}^{-l / 2}\right]>L_{p}\left[\bar{y}^{l / 2}\right]$.

Suppose that we are in case (a). Then $\widetilde{\sigma}^{-l_{0}-1}\left[\bar{y}^{l / 2}\right]$ is the center of some $p$-bridge which contains $\tilde{\sigma}^{-l_{0}-1}\left[\bar{y}^{-l / 2}\right]$, and by the same argument as given in case (1) we see that $L_{p}\left[\bar{y}^{l}\right]=L_{p}\left[\bar{y}^{2 l}\right]$. This again yields a contradiction.

Suppose that we are in case (b). Then $\tilde{\sigma}^{-l_{0}-1}\left[\bar{y}^{-l / 2}\right]$ is now the center of a $p$-bridge which contains $\tilde{\sigma}^{-l_{0}-1}\left[\bar{y}^{l / 2}\right]$. Since $L_{p}\left[\bar{y}^{l / 2+i}\right]<L_{p}\left[\bar{y}^{l / 2}\right]$ for all $i \in\{1, \ldots, l / 2+a\}$, this $p$-bridge will also contain $\tilde{\sigma}^{-l_{0}-1}\left[\bar{y}^{l+a}\right]$. So the boundary points of this $p$-bridge extend past $\tilde{\sigma}^{-l_{0}-1}\left[\bar{y}^{-2 l-a}\right]$. Since the center of the symmetry of the $\tilde{\sigma}^{l_{0}+1}$ image of this bridge is $\left[\bar{y}^{-l / 2}\right]$ we see that $L_{p}\left[\bar{y}^{-2 l}\right]=L_{p}\left[\bar{y}^{l}\right]$. By considering $h_{p, g}^{\prime}$ of this arc we will reach the same contradiction as in case (1) but this time with $\left[\bar{y}^{\prime}-2 d\right]$ and $\left[\bar{y}^{\prime d}\right]$.

Note that the above proof also shows that the following corollary holds:

Corollary 4.10 For every quasi-q-symmetric arc $A^{\prime}$ of $C^{\prime}$, the $\operatorname{arc} h_{q, p}\left(A^{\prime}\right)$ is quasi-p-symmetric.

Acknowledgements The authors are grateful to the referee for useful remarks and comments which enabled them to improve the presentation of the paper.

The research of B E Raines is supported by the NSF grant 0604958. The research of S Štimac is supported in part by the NSF grant 0604958 and in part by the MZOS grant 037-0372791-2802 of the Republic of Croatia.

\section{References}

[1] J E Anderson, IF Putnam, Topological invariants for substitution tilings and their associated $C^{*}$-algebras, Ergodic Theory Dynam. Systems 18 (1998) 509-537 MR1631708

[2] M Barge, K Brucks, B Diamond, Self-similarity in inverse limit spaces of the tent family, Proc. Amer. Math. Soc. 124 (1996) 3563-3570 MR1363409 
[3] M Barge, B Diamond, The dynamics of continuous maps of finite graphs through inverse limits, Trans. Amer. Math. Soc. 344 (1994) 773-790 MR1236222

[4] M Barge, B Diamond, Homeomorphisms of inverse limit spaces of one-dimensional maps, Fund. Math. 146 (1995) 171-187 MR1314982

[5] M Barge, B Diamond, A complete invariant for the topology of one-dimensional substitution tiling spaces, Ergodic Theory Dynam. Systems 21 (2001) 1333-1358 MR1855835

[6] M Barge, J Jacklitch, G Vago, Homeomorphisms of one-dimensional inverse limits with applications to substitution tilings, unstable manifolds, and tent maps, from: "Geometry and topology in dynamics (Winston-Salem, NC, 1998/San Antonio, TX, 1999)", (M Barge, K Kuperberg, editors), Contemp. Math. 246, Amer. Math. Soc. (1999) 1-15 MR1732368

[7] M Barge, J Martin, Chaos, periodicity, and snakelike continua, Trans. Amer. Math. Soc. 289 (1985) 355-365 MR779069

[8] M Barge, J Martin, The construction of global attractors, Proc. Amer. Math. Soc. 110 (1990) 523-525 MR1023342

[9] M Barge, J Martin, Endpoints of inverse limit spaces and dynamics, from: "Continua (Cincinnati, OH, 1994)", (H Cook, W T Ingram, K T Kuperberg, A Lelek, P Minc, editors), Lecture Notes in Pure and Appl. Math. 170, Dekker, New York (1995) 165-182 MR1326840

[10] L Block, S Jakimovik, L Kailhofer, J Keesling, On the classification of inverse limits of tent maps, Fund. Math. 187 (2005) 171-192 MR2214878

[11] K Brucks, H Bruin, Subcontinua of inverse limit spaces of unimodal maps, Fund. Math. 160 (1999) 219-246 MR1708988

[12] K M Brucks, B Diamond, A symbolic representation of inverse limit spaces for a class of unimodal maps, from: "Continua (Cincinnati, OH, 1994)", (H Cook, W T Ingram, K T Kuperberg, A Lelek, P Minc, editors), Lecture Notes in Pure and Appl. Math. 170, Dekker, New York (1995) 207-226 MR1326844

[13] H Bruin, Planar embeddings of inverse limit spaces of unimodal maps, Topology Appl. 96 (1999) 191-208 MR1709688

[14] H Bruin, Inverse limit spaces of post-critically finite tent maps, Fund. Math. 165 (2000) 125-138 MR1808727

[15] H Bruin, Subcontinua of Fibonacci-like inverse limit spaces, Topology Proc. 31 (2007) 37-50 MR2363150

[16] P Collet, J-P Eckmann, Iterated maps on the interval as dynamical systems, Progr. Physics 1, Birkhäuser, Mass. (1980) MR613981 
[17] C Good, R Knight, B E Raines, Nonhyperbolic one-dimensional invariant sets with a countably infinite collection of inhomogeneities, Fund. Math. 192 (2006) 267-289 MR2278390

[18] C Good, B E Raines, Continuum many tent map inverse limits with homeomorphic postcritical $\omega$-limit sets, Fund. Math. 191 (2006) 1-21 MR2232193

[19] L Kailhofer, A partial classification of inverse limit spaces of tent maps with periodic critical points, Topology Appl. 123 (2002) 235-265 MR1918499

[20] L Kailhofer, A classification of inverse limit spaces of tent maps with periodic critical points, Fund. Math. 177 (2003) 95-120 MR1992527

[21] J Kennedy, D R Stockman, J A Yorke, Inverse limits and models with backward dynamics, to appear in Journal Math. Econ.

[22] J Kennedy, D R Stockman, J A Yorke, Inverse limits and an implicitly defined difference equation from economics, Topology Appl. 154 (2007) 2533-2552 MR2332869

[23] A Medio, B E Raines, Inverse limit spaces arising from problems in economics, Topology Appl. 153 (2006) 3437-3449 MR2270597

[24] A Medio, B E Raines, Backward dynamics in economics. The inverse limit approach, J. Econom. Dynam. Control 31 (2007) 1633-1671 MR2317572

[25] B E Raines, Inhomogeneities in non-hyperbolic one-dimensional invariant sets, Fund. Math. 182 (2004) 241-268 MR2098780

[26] B E Raines, S Štimac, Structure of inverse limit spaces of tent maps with nonrecurrent critical points, Glas. Mat. Ser. III 42(62) (2007) 43-56 MR2332656

[27] S Štimac, Topological classification of Knaster continua with finitely many endpoints (Croatian), PhD thesis, University of Zagreb (2002)

[28] S Štimac, Structure of inverse limit spaces of tent maps with finite critical orbit, Fund. Math. 191 (2006) 125-150 MR2231059

[29] S Štimac, A classification of inverse limit spaces of tent maps with finite critical orbit, Topology Appl. 154 (2007) 2265-2281 MR2328011

[30] R Swanson, H Volkmer, Invariants of weak equivalence in primitive matrices, Ergodic Theory Dynam. Systems 20 (2000) 611-626 MR1756989

Department of Mathematics, Baylor University

Waco, TX, USA

Graduate School of Economics and Business, University of Zagreb

Kennedyev trg 6, 10000 Zagreb, Croatia

brian_raines@baylor.edu, sonja@math.hr

http: //www . baylor . edu/math/index . php?id=54016, http://www.efzg.hr/sstimac

Received: 7 January $2008 \quad$ Revised: 11 April 2009 